\title{
Prophage-encoded gene VpaChn25_0734 amplifies ecological persistence of Vibrio parahaemolyticus CHN25
}

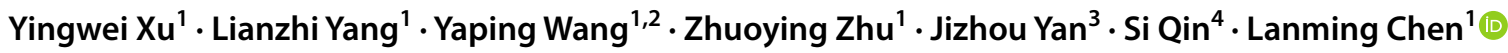

Received: 3 December 2021 / Revised: 3 December 2021 / Accepted: 31 December 2021 / Published online: 22 January 2022

(c) The Author(s) 2022

\begin{abstract}
Vibrio parahaemolyticus is a waterborne pathogen that can cause acute gastroenteritis, wound infection, and septicemia in humans. The molecular basis of its pathogenicity is not yet fully understood. Phages are found most abundantly in aquatic environments and play a critical role in horizontal gene transfer. Nevertheless, current literature on biological roles of prophage-encoded genes remaining in $V$. parahaemolyticus is rare. In this study, we characterized one such gene VpaChn25_0734 (543-bp) in V. parahaemolyticus CHN25 genome. A deletion mutant $\Delta$ VpaChn25_0734 (543-bp) was obtained by homologous recombination, and a revertant $\Delta V p a C h n 25 \_0734-c o m$ (543-bp) was also constructed. The $\Delta$ VpaChn25_0734 (543-bp) mutant was defective in growth and swimming mobility particularly at lower temperatures and/ or pH 7.0-8.5. Cell surface hydrophobicity and biofilm formation were significantly decreased in the $\Delta$ VpaChn25_0734 (543-bp) mutant $(p<0.05)$. Based on the in vitro Caco-2 cell model, the deletion of VpaChn25_0734 (543-bp) gene significantly reduced the cytotoxicity of $V$. parahaemolyticus CHN25 to human intestinal epithelial cells $(p<0.05)$. Comparative secretomic and transcriptomic analyses revealed a slightly increased extracellular proteins, and thirteen significantly changed metabolic pathways in the $\triangle V p a C h n 25 \_0734$ (543-bp) mutant, showing down-regulated carbon source transport and utilization, biofilm formation, and type II secretion system $(p<0.05)$, consistent with the observed defective phenotypes. Taken, the prophage-encoded gene VpaChn25_0734 (543-bp) enhanced V. parahaemolyticus CHN25 fitness for survival in the environment and the host. The results in this study facilitate better understanding of pathogenesis and genome evolution of V. parahaemolyticus, the leading sea foodborne pathogen worldwide.
\end{abstract}

Keywords Vibrio parahaemolyticus · Foodborne pathogen · Prophage · Gene deletion and reversion · Secretome . Transcriptome

\section{Introduction}

Vibrio parahaemolyticus is a Gram-negative bacterium that resides in aquatic environments worldwide (Letchumanan et al. 2014; Su and Chen 2020). Ingestion of raw,

Communicated by Michael Polymenis.

Si Qin

qinsiman@hunau.edu.cn

$\triangle$ Lanming Chen

lmchen@shou.edu.cn

1 Key Laboratory of Quality and Safety Risk Assessment for Aquatic Products on Storage and Preservation (Shanghai), Ministry of Agriculture and Rural Affairs of the People's Republic of China, College of Food Science and Technology, Shanghai Ocean University, Shanghai 201306, China undercooked, or mishandled seafood contaminated by pathogenic $V$. parahaemolyticus can cause acute gastroenteritis in humans and even death (Ghenem et al. 2017; Baker-Austin et al. 2018). The bacterium was first identified from semidried juvenile sardines in 1950 in Japan, which caused 272 cases of acute diarrhea and 20 death (Fujino et al. 1953).

2 Department of Internal Medicine, Virginia Commonwealth University/McGuire VA Medical Centre, Richmond, VA, USA

3 College of Fishers and Life Science, Shanghai Ocean University, Shanghai 201306, China

4 Key Laboratory for Food Science and Biotechnology of Hunan Province, College of Food Science and Technology, Hunan Agricultural University, Changsha 410128, China 
Afterward, outbreaks of human gastroenteritis caused by $V$. parahaemolyticus occurred in many Asian nations and consequently around the world (Elmahdi et al. 2016; Meparambu Prabhakaran et al. 2020). It was estimated that more than $50 \%$ of foodborne gastrointestinal-Vibriosis cases were caused by V. parahaemolyticus in the United States (Karan et al. 2021), which led to 45,000 illnesses each year (https:// www.cdc.gov/Vibrio/, accessed on 26 April 2021). Sporadic outbreaks have also been reported in coastal European nations (Meparambu Prabhakaran et al. 2020). China is one of the 15 countries with high incidence of diarrhea disease (Walker et al. 2013). Recently, a national surveillance of 152,792 patients of all ages with acute diarrhea was administered in 217 hospitals and 93 reference laboratories in 31 provinces of China by the Chinese Center for Diseases Control and Prevention in 2009-2018 (Wang et al. 2021a). Total 13 common bacterial pathogens were tested, among which $V$. parahaemolyticus ranked third and contributed to $10.83 \%$ of all positive detection. Pathogenic V. parahaemolyticus strains produce thermostable direct hemolysin (TDH) and/ or the TDH-related hemolysin (TRH) (Leoni et al. 2016; Cai and Zhang 2018). However, some clinical V. parahaemolyticus strains do not produce the two major toxins but display virulence, indicating that putative toxic determinants exist. Their pathogenicity might be achieved with different strategies employed by different strains ( $\mathrm{Li}$ et al. 2020). Therefore, it is imperative to identify such risk factors in V. parahaemolyticus to assure food safety and human health.

Phages are found most abundantly in aquatic environments worldwide (Tan et al. 2021). Temperate phages can integrate into bacterial chromosomes with carried genes and drive bacterial genome evolution (Garin-Fernandez and Wichels 2020; Nuidate et al. 2021; Wendling et al. 2021). Vibrio spp. virulence-associated genes can be combined at high frequencies by horizontal gene transfer (HGT), leading to the emergence of pandemic or pathogenic clones (Castillo et al. 2018). For example, in early 1996, an atypical outbreak of $V$. parahaemolyticus infection in India was associated with a new serotype O3:K6 that carried genetic markers $t d h$, tox $R S_{\text {new }}$, and orf8. Dispersal of this new pathogenic clone has been reported in many countries around five continents, suggesting that $V$. parahaemolyticus from the serotype O3:K6 was pandemic (Santos et al. 2021). The orf8 gene was possibly acquired from a filamentous phage f237, which endorsed the bacterium more adhesive to host intestinal cells (Santos et al. 2021). Recently, Grain-Fernandez et al. reported a filamentous phage vB_VpaI_VP-3218 in $V$. parahaemolyticus $\mathrm{VN}-3218$. It integrates into the host genome with potential zonula occludens toxin genes (GarinFernandez et al. 2020). Another newly isolated phage $\mathrm{vB}_{-}$ VpaP_MGD2 from clam Meretrix meretrix was reported to be a candidate biocontrol agent against early mortality syndrome/acute hepatopancreatic necrosis disease caused by multidrug-resistant $V$. parahaemolyticus in shrimp (Cao et al. 2021).

In our previous research, V. parahaemolyticus CHN25 (serotype: O5: KUT) of aquatic animal origin was identified and characterized (Song et al. 2013; Sun et al. 2014; He et al. 2015; Zhu et al. 2017, 2020; Yang et al. 2020). The bacterial genome $(5,443,401 \mathrm{bp}, 45.2 \% \mathrm{G}+\mathrm{C})$ contains five prophage gene clusters (6.5-36.6 kb) (Zhu et al. 2017), the largest of which showed sequence similarity to a Vibrio phage Martha 12B12 (Zhu et al. 2017). Within the cluster, a gene VpaChn25_0724 (294-bp) encoding a hypothetical protein has been identified recently (Yang et al. 2020). In the present study, biological function of another prophageencoded gene VpaChn25_0734 (543-bp) within the same gene cluster was characterized. It showed sequence identity with components of a Phage_tail_S superfamily (Lu et al. 2020). The objectives of this study were (1) to knock out the VpaChn25_0734 (543-bp) gene from V. parahaemolyticus CHN25 genome by homologous recombination to contract a deletion mutant $\Delta$ VpaChn25_0734 (543-bp). Meanwhile, a revertant $\Delta V p a C h n 25 \_0734$-com (543-bp) was also constructed; (2) to examine growth, mobility, biofilm formation, and cell toxicity of the $\Delta V p a C h n 25 \_0734$ (543-bp) mutant compared with $V$. parahaemolyticus CHN25 wild type (WT) and $\Delta$ VpaChn25_0734-com (543-bp) strains; (3) to decipher the possible molecular mechanism underlying altered phenotypes of the $\Delta$ VpaChn25_0734 (543-bp) mutant by comparative secretomic and transcriptomic analysis. The results in this study facilitate better understanding of biological function of prophage-encoded genes in V. parahaemolyticus genomes.

\section{Materials and methods}

\section{Bacterial strains, plasmids, and culture conditions}

Vibrio parahaemolyticus CHN25 (Song et al. 2013; Sun et al. 2014; He et al. 2015; Zhu et al. 2017, 2020; Yang et al. 2020) was stored at $-80{ }^{\circ} \mathrm{C}$ freezer in our research group. Escherichia coli DH5 $\alpha \lambda$ pir (BEINUO Biotech (Shanghai) Co. Ltd., China), E. coli $\beta 2155 \lambda$ pir (kindly provided by Professor Weicheng Bei) and plasmid pDS132 (kindly provided by Professor Dominique Schneider) were used as a host strain for gene cloning, and a donor strain and a suicide vector for conjugation and gene knockout experiments, respectively (Zhu et al. 2017; Yang et al. 2020). Plasmid pMMB207 (Biovector Science Lab, Inc., China) was used as a gene expression vector (Zhu et al. 2017; Yang et al. 2020). $V$. parahaemolyticus and E. coli strains were incubated in the same media and conditions as described previously (Zhu et al. 2017; Yang et al. 2020). 


\section{Construction of the gene deletion mutant and revertant}

Gene deletion mutant and revertant of $V$. parahaemolyticus CHN25 were constructed according to the methods described previously (Zhu et al. 2017; Yang et al. 2020). Briefly, two primer pairs VpaChn25_0734 (543-bp)-upF/R and VpaChn25_0734 (543-bp)-down-F/R (Table 1) were designed to target upstream (486 bp) and downstream (494 bp) sequences of the VpaChn25_0734 (543-bp) gene in $V$. parahaemolyticus CHN25 genome, respectively. Restriction endonucleases XbaI and SacI (TaKaRa, Japan) were introduced into polymerase chain reaction (PCR) products, which were inserted into corresponding cloning sites of the pDS132. The obtained recombinant plasmid pDS132 + VpaChn25_0734 (543-bp) was then transformed into diaminopimelic acid (DAP) auxotroph E. coli $\beta 2155$ incubated in LB medium supplemented with $0.3 \mathrm{mM}$ DAP (Sigma-Aldrich, USA) (Zhu et al. 2017; Yang et al. 2020). Double-crossover deletions of the VpaChn25_0734 (543-bp) gene were screened using the colony PCR assay (Zhu et al. 2017; Yang et al. 2020) with the primer pair
VpaChn25_0734 (543-bp)-up-ex-F/R (Table 1). The obtained $\Delta$ VpaChn25_0734 (543-bp) mutant was confirmed as described previously (Zhu et al. 2017; Yang et al. 2020).

Additionally, the VpaChn25_0734 (543-bp) gene was obtained by PCR using the primer pair VpaChn25_0734com (543-bp)-F/-R (Table 1). The PCR product was inserted into the pMMB207, and then transformed into $E$. coli $\mathrm{DH} 5 \alpha$. The obtained recombinant plasmid pMMB207 + VpaChn25_0734 (543-bp) was electro-transformed into the $\Delta$ VpaChn25_0734 (543-bp) mutant (Zhu et al. 2017; Yang et al. 2020). Positive electro-transformants $\Delta$ VpaChn25_0734-com (543-bp) were screened, and confirmed (Zhu et al. 2017; Yang et al. 2020). DNA sequencing was conducted by Shanghai Sangon Biological Engineering Technology and Services Co., Ltd., Shanghai, China.

\section{Growth curve assay}

Growth of $V$. parahaemolyticus strains in the LB or TSB media at different temperatures $\left(37^{\circ} \mathrm{C}, 25^{\circ} \mathrm{C}\right.$, and $\left.15{ }^{\circ} \mathrm{C}\right)$ for different time (24-60 h) was individually measured using Bioscreen Automated Growth Curve Analyzer (BioTek,

Table 1 Oligonucleotide primers used in this study

\begin{tabular}{|c|c|c|c|}
\hline Primer & Sequence $\left(5^{\prime}->3^{\prime}\right)$ & Product size (bp) & References \\
\hline VpaChn25_0734 (543-bp)-up-F & GCTCTAGATGTGATCGACATCGAACAAC & 486 & This study \\
\hline VpaChn25_0734 (543-bp)-up-R & CTCATGGCAGTTGAGCCATCAAAGCACTCC & & \\
\hline VpaChn25_0734 (543-bp)-down-F & GATGGCTCAACTGCCATGAGCCGCCCCGAT & 494 & This study \\
\hline VpaChn25_0734 (543-bp)-down-R & CGAGCTC CTCTTCATCGAGATACCATTG & & \\
\hline VpaChn25_0734 (543-bp)-up-ex-F & CGGCGCTCTTAAAGTTCGCTC & 525 & This study \\
\hline VpaChn25_0734 (543-bp)-down-ex-R & GCGCGACTTCTTCTCCGTC & & \\
\hline VpaChn25_0734-com (543-bp)-F & CGAGCTCATGGCTCAAGC GGTTCGC & 543 & This study \\
\hline VpaChn25_0734-com (543-bp)-R & GCTCTAGATCATGGCAGA AGCTCCTT & & \\
\hline $\operatorname{tlh}-F$ & AAAGCGGATTATGCAGAAGCACTG & 596 & Yang et al. (2020) \\
\hline$t l h-R$ & ACTTTCTAGCATTTTCTCTGC & & \\
\hline 16s $R N A-F$ & GACACGGTCCAGACTCCTAC & 179 & Yang et al. (2020) \\
\hline 16s $R N A-R$ & GGTGCTTCTTCTGTCGCTAAC & & \\
\hline VpaChn25_0734 (543-bp)-F & CCGGAATTC ATGGCTCAAGCGGTTCGC & 543 & This study \\
\hline VpaChn25_0734 (543-bp)-R & CCGCTCGAGTCATGGCAGAAGCTCCTT & & \\
\hline VpaChn25_RSO2080-F & TGCTTGGGATAAAGACGA & 1365 & This study \\
\hline VpaChn25_RSO2080-R & GTAAACGGCGTGACTTCT & & \\
\hline VpaChn25_RSO3685-F & TTGTAGCAGCCATGAAAG & 873 & This study \\
\hline VpaChn25_RS03684-R & TATTGCGACAGCGGATAT & & \\
\hline VpaChn25_RS10950-F & ATGAAGACGGATTGGATT & 1512 & This study \\
\hline VpaChn25_RS10950-R & GTGAAAGAGGAGTTGGGT & & \\
\hline VpaChn25_RS11345-F & GGTGTTGCTTGCCGTTAG & 756 & This study \\
\hline VpaChn25_RS11345-R & CCGGAGGAAGAAGTTGGT & & \\
\hline VpaChn25_RS13840-F & TGATGAGACGCACAAATA & 873 & This study \\
\hline VpaChn25_RS13840-R & GTAAGGATGTAACGACCAA & & \\
\hline VpaChn25_RS14605-F & TTTCACGCACTTCCACTT & 963 & This study \\
\hline VpaChn25_RS14605-R & TACAGCACTATCCGAGGG & & \\
\hline
\end{tabular}


USA). Growth curves of $V$. parahaemolyticus strains in the TSB medium in different $\mathrm{pH}$ conditions ( $\mathrm{pH} 5.5-8.5$ ) were also examined, respectively (Sun et al. 2014; Zhu et al. 2017; Yang et al. 2020).

\section{Swimming motility and biofilm formation assays}

Swimming motility of $V$. parahaemolyticus strains on semisolid TSB (pH 8.5, 3\% NaCl) agar plates $(0.25 \%$ agar) was individually measured as described previously (Yang et al. 2020). The TSB agar plates were incubated at $37^{\circ} \mathrm{C}, 25^{\circ} \mathrm{C}$, and $15{ }^{\circ} \mathrm{C}$ for $12 \mathrm{~h}, 24 \mathrm{~h}$, and $72 \mathrm{~h}$, respectively. Diameters at bacterial colonies were measured and recorded. Biofilm formation of $V$. parahaemolyticus strains was individually examined using the crystalline violet staining method (Yang et al. 2020). The phosphate-buffered saline (PBS, pH 7.2-7.4), and crystal violet were purchased from Sangon (China).

\section{Bacterial cell membrane permeability and fluidity, and surface hydrophobicity assays}

Outer membrane permeability was determined according to the method by Wang et al. (Wang et al. 2021c). Briefly, a $200 \mu \mathrm{L} /$ well of bacterial suspension in TSB medium ( $\mathrm{pH}$ $8.5,3 \% \mathrm{NaCl}$ ) at mid-logarithmic growth phase (mid-LGP) at $37^{\circ} \mathrm{C}$ was mixed with a $2 \mu \mathrm{L} /$ well of $10 \mathrm{~mm} \mathrm{~N}$-phenyl1-naphthylamine (NPN) (Sangon, China). The change of fluorescence intensity per well was measured using BioTek Synergy 2 (BioTek, USA). The excitation and emission wavelengths were $350 \mathrm{~nm}$ and $420 \mathrm{~nm}$, respectively (Wang et al. 2021c). Inner membrane permeability was also determined (Huang et al. 2021). Briefly, a $200 \mu \mathrm{L} /$ well of bacterial suspension was mixed with a $2.5 \mu \mathrm{L} /$ well of $10 \mathrm{mM}$ $O$-nitrophenyl- $\beta$-D-galactopyranoside $(O$-nitrophenyl)- $\beta$-Dgalactopyranoside (ONPG) (Sangon, China). The mixture was incubated at $37^{\circ} \mathrm{C}$, and absorbance at $\mathrm{OD}_{415 \mathrm{~nm}}$ of each well was measured using BioTek synergy 2 (BioTek, USA) every 30 min (Huang et al. 2021). Cell surface hydrophobicity and membrane fluidity assays were performed as described previously (Yang et al. 2020).

\section{Secretome analysis}

Extraction of extracellular proteins of $V$. parahaemolyticus strains grown in TSB medium $(\mathrm{pH} 8.5,3 \% \mathrm{NaCl})$ at $37{ }^{\circ} \mathrm{C}$ in static culture condition, and two-dimensional gel electrophoresis (2D-GE) were performed according to the methods described previously (Zhu et al. 2020). Briefly, a $20 \mathrm{mg}$ of each sample was applied to $\mathrm{pH}$ gradient gel strips ( $\mathrm{pH} 4-7$, $7 \mathrm{~cm}$, Bio-Rad, Inc., USA) at $17^{\circ} \mathrm{C}$ for passive rehydration for $16 \mathrm{~h}$. Isoelectric focusing (IEF) for the first dimensional separation was performed using a six-step procedure, while sodium dodecyl sulfate-polyacrylamide gel electrophoresis (SDS-PAGE, 12.5\% separation gels) was performed for the second dimensional separation (Zhu et al. 2020). Amino acid sequences of protein spots were determined using liquid chromatography-tandem mass spectrometry (LC-MS/ MS) by Shanghai Houji Biological Co., Shanghai, China (Zhu et al. 2020).

\section{Human intestinal epithelial cell viability and apoptosis assay}

The in vitro cell modal assay was performed according to the method described previously [26], using human rectal cancer epithelial cell line Caco-2 (ATCC number: HTB$37^{\mathrm{TM}}$, Stem Cell Bank of Chinese Academy of Sciences, Shanghai, China). Briefly, Caco- 2 cells $\left(5 \times 10^{4}\right.$ cells $/ \mathrm{mL}$ per well) were cultured in Dulbecco's modified eagle medium (DMEM, Gibco, USA) at $37{ }^{\circ} \mathrm{C}$ with $5 \% \mathrm{CO}_{2}$ for $24 \mathrm{~h}$, and then washed three times with 0.1 M PBS (pH 7.2-7.4, Sangon, China). Each of V. parahaemolyticus strains was grown in TSB medium (pH 8.5, $3 \% \mathrm{NaCl}$ ) at $37^{\circ} \mathrm{C}$ to mid-LGP, and then harvested, washed, and resuspended in DMEM medium without phenol red. A $100 \mu \mathrm{L} /$ well of bacterial suspension $\left(\mathrm{OD}_{490 \mathrm{~nm}}\right.$ of about $\left.0.2 \pm 0.02\right)$, and $10 \mu \mathrm{L} /$ well of 2-(2-methoxy-4-nitrophenyl)-3-(4-nitrophenyl)-5-(2,4-disulfonate)2h-tetrazolium monosodium salt (CCK-8, Sigma-Aldrich, USA) were added into Caco- 2 cells per well, and then incubated at $37^{\circ} \mathrm{C}$ for $4 \mathrm{~h}$. Caco- 2 cell viability and apoptosis were examined using Annexin-V-FITC/PI Apoptosis Assay Kit (Solarbio, China) and BD FACSVerse ${ }^{\mathrm{TM}}$ flow cytometer (Becton, Dickinson and Company, USA) according to the instructions of the manufacturers (Yang et al. 2020).

\section{Transcriptome and data analysis}

Total RNA of each of V. parahaemolyticus strains grown in TSB medium ( $\mathrm{pH} 8.5,3 \% \mathrm{NaCl}$ ) to mid-LGP at $37^{\circ} \mathrm{C}$ were individually extracted, and purified as described previously (Yang et al. 2020). Three independently prepared RNA samples were subjected for Illumina RNA-sequencing, which was carried out by Shanghai Majorbio Bio-pharm Technology Co. Ltd., China using Illumina HiSeq 2500 platform (Yang et al. 2020). Quality filtration of raw RNA-seq data, clean read aligning, and defining of differentially expressed genes (DEGs) were conducted as described previously (Yang et al. 2020). Gene set enrichment analysis (GSEA) of DEGs was also conducted (Yang et al. 2020). Representative DEGs were examined using quantitative reverse transcription-PCR (RT-PCR) assay (Yang et al. 2020) with the primers listed in Table 1.

The data were analyzed using SPSS version 17.0 software (SPSS Inc., USA). All tests in this study were performed in 
at least triplicate. Phylogenetic tree was constructed using MEGA 5.0 software (Kumar et al. 2018).

\section{Scanning electron microscopy (SEM) analysis}

Cell structure of $V$. parahaemolyticus strains grown in TSB medium ( $\mathrm{pH} 8.5,3 \% \mathrm{NaCl})$ to mid-LGP at $37{ }^{\circ} \mathrm{C}$ were observed and recorded using Schottky Field Emission Scanning Electron Microscope (SU5000, Japan, $5.0 \mathrm{kV} \times 35,000$ ) at College of Food Science and Technology, Shanghai Ocean University (Shanghai, China).

\section{Results}

\section{Prophage-encoded gene VpaChn25_0734 (543-bp) in V. parahaemolyticus CHN25}

The largest prophage gene cluster contains 24 predicted open read frames (ORFs) in V. parahaemolyticus CHN25 chromosome 1 (3,416,467 bp, from 816,554 to $846,961 \mathrm{bp})$ (Zhu et al. 2017). It has sequence similarity to the Vibrio phage Martha 12B12 (30,408 bp, GenBank accession no. NC_021070) (Yang et al. 2020). The VpaChn25_0734 (543bp) gene is the sixteenth ORF encodes a predicted phage virion morphogenetic protein with conserved structural domains belonging to the Phage_tail_S superfamily. In the cellular component catalogue, it was involved in constituting the intracellular membrane-bounded organelle (GO: 0043231) with a predicted GO-Score of 0.42 .

\section{Deletion and reverse complementation of the VpaChn25_0734 (543-bp) gene}

To characterize biological roles of the prophageencoded gene VpaChn25_0734 (543-bp) remaining in $V$. parahaemolyticus $\mathrm{CHN} 25$ genome, an untagged in-frame gene deletion mutant $\Delta$ VpaChn25_0734 (543-bp) was obtained by homologous recombination (see the Materials and Methods). The VpaChn25_0734 (543-bp) gene was knocked out from the $V$. parahaemolyticus CHN25 genome, which was verified by PCR and DNA sequencing (data not shown), RT-qPCR, as well as transcriptome analysis (see below).

Additionally, a reverse mutant $\Delta$ VpaChn25_0734com (543-bp) was also successfully obtained. The VpaChn25_0734 (543-bp) gene was amplified, and cloned into the expression vector pMMB207, resulting in the recombinant vector pMMB207 + VpaChn25_0734 (543-bp). This recombinant vector was electro-transformed into the $\Delta$ VpaChn25_0734 (543-bp) mutant to obtain the revertant $\Delta$ VpaChn25_0734-com (543-bp), which was confirmed by the methods described above.

\section{Survival of $\triangle$ VpaChn25_0734 (543-bp) at different temperatures and $\mathrm{pH}$ conditions}

To investigate the influence of VpaChn25_0734 (543-bp) gene deletion on $V$. parahaemolyticus $\mathrm{CHN} 25$ survival, we determined growth curves of the WT, $\triangle V p a C h n 25 \_0734$ (543-bp), and $\Delta$ VpaChn25_0734-com (543-bp) strains at $37{ }^{\circ} \mathrm{C}, 25{ }^{\circ} \mathrm{C}$, and $15{ }^{\circ} \mathrm{C}$, which $V$. parahaemolyticus experiences during life cycle (Maje et al. 2020; Yang et al. 2020; Ali et al. 2021). At the optional growth temperature of $37^{\circ} \mathrm{C}$, the $\Delta$ VpaChn25_0734 (543-bp) mutant grew in TSB medium $(\mathrm{pH} 8.5,3 \% \mathrm{NaCl})$ with a retardation phase (RP) of $2 \mathrm{~h}$, when compared with the WT strain (Fig. 1a). Similarly, at $25{ }^{\circ} \mathrm{C}$ and $15{ }^{\circ} \mathrm{C}$, the RPs of $\Delta$ VpaChn25_0734 (543-bp) were sixfold and 2.67-fold longer than those of WT, respectively (Fig. 1b, c). Additionally, the revertant $\Delta$ VpaChn25_0734-com (543-bp) appeared partially complement the defective phenotype of
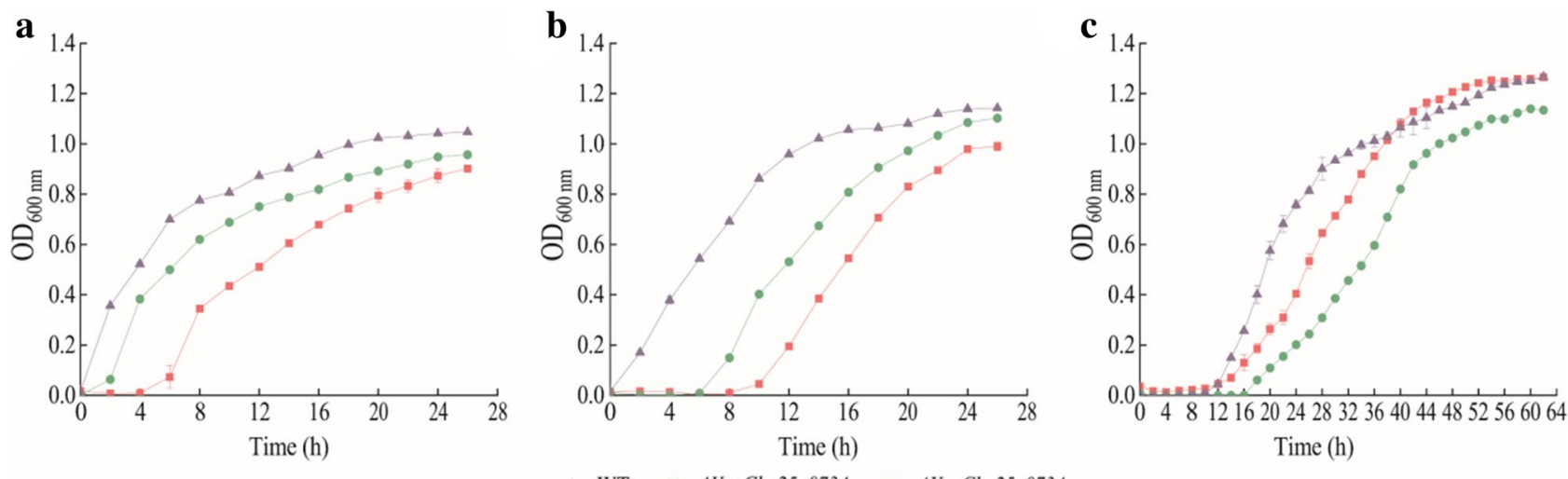

Fig. 1 Survival of V. parahaemolyticus CHN25 (WT), AVpaChn25_0734 (543-bp), and AVpaChn25_0734-com (543-bp) strains at different temperatures. a-c $37{ }^{\circ} \mathrm{C}(\mathbf{a}), 25^{\circ} \mathrm{C}(\mathbf{b})$, and $15^{\circ} \mathrm{C}(\mathbf{c})$, respectively 
the $\Delta$ VpaChn25_0734 (543-bp) mutant. These results indicated that the VpaChn25_0734 (543-bp) gene enhanced V. parahaemolyticus $\mathrm{CHN} 25$ fitness for thriving particularly at lower temperatures.

Acid tolerance is critical for foodborne pathogens to survive against host acid gastric fluid ( $\mathrm{pH} \mathrm{1-3}$, but above 6.0 after food consumption) (Sun et al. 2014). Therefore, we examined the growth of the three strains in TSB medium (3\% $\mathrm{NaCl}$ ) with $\mathrm{pH}$ values in the range from 5.5 to 8.0 , and the results were illustrated in Fig. 2a-f. In acidic conditions (pH 5.5-6.5), the growth of WT, $\Delta V p a C h n 25 \_0734$ (543-bp), and $\Delta V p a C h n 25 \_0734$-com (543-bp) strains were greatly inhibited, and the maximum $\mathrm{OD}_{600 \mathrm{~nm}}$ values at stationary grow phage (SGP) were below 0.5 (Fig. 2a-c); in the neutral condition (pH 7.0), the growth of $\Delta$ VpaChn25_0734 (543bp) and $\Delta V p a C h n 25 \_0734$-com (543-bp) strains were also obviously inhibited compared to the WT strain (Fig. 2d); in alkaline conditions ( $\mathrm{pH}$ 7.5-8.0), $\Delta$ VpaChn25_0734 (543-bp) still grew poorly with a longer RP and lower biomass than WT (Fig. 2e, f). Additionally, the plasmid-born $\Delta$ VpaChn25_0734-com (543-bp) did not fully complement the defective phenotype of the $\Delta V p a C h n 25 \_0734$ (543-bp) mutant. The results indicated that the VpaChn25_0734 (543-bp) gene amplified $V$. parahaemolyticus CHN25 environmental persistance at $\mathrm{pH}$ 7.0-8.0.

\section{Swimming mobility of the $\triangle V p a C h n 25 \_0734$ (543-bp) mutant}

Vibrio parahaemolyticus is found to grow as swimming cells in liquid environments (Freitas et al. 2020). In this study, swimming mobility of the WT, $\triangle$ VpaChn25_0734 (543-bp), and $\Delta V p a C h n 25 \_0734-c o m$ (543-bp) strains were examined at different temperatures. As shown in Fig. 3a-1 to $\mathrm{c}-1$, no significant difference in swimming circles was observed among the three strains grown in semi-solid TSB agar medium ( $\mathrm{pH} 8.5,3 \% \mathrm{NaCl}, 0.25 \%$ agar) at $37{ }^{\circ} \mathrm{C}$ ( $p>0.05$ ). However, at $25^{\circ} \mathrm{C}$, the $\Delta V p a C h n 25 \_0734$ (543bp) mutant swam significantly slower $(8.00 \pm 0.71 \mathrm{~mm})$ than the WT $(12.75 \pm 2.86 \mathrm{~mm})$, and $\Delta V p a C h n 25 \_0734$-com (543-bp) $(9.00 \pm 1.2 \mathrm{~mm})$ strains, respectively $(p<0.05)$ (Fig. 3a-2 to c-2). The similar case was observed at $15{ }^{\circ} \mathrm{C}$ (Fig. 3a-3 to c-3). These results indicated that the deletion of VpaChn25_0734 (543-bp) gene significantly inhibited swimming mobility of $V$. parahaemolyticus $\mathrm{CHN} 25$ at the lower temperatures.
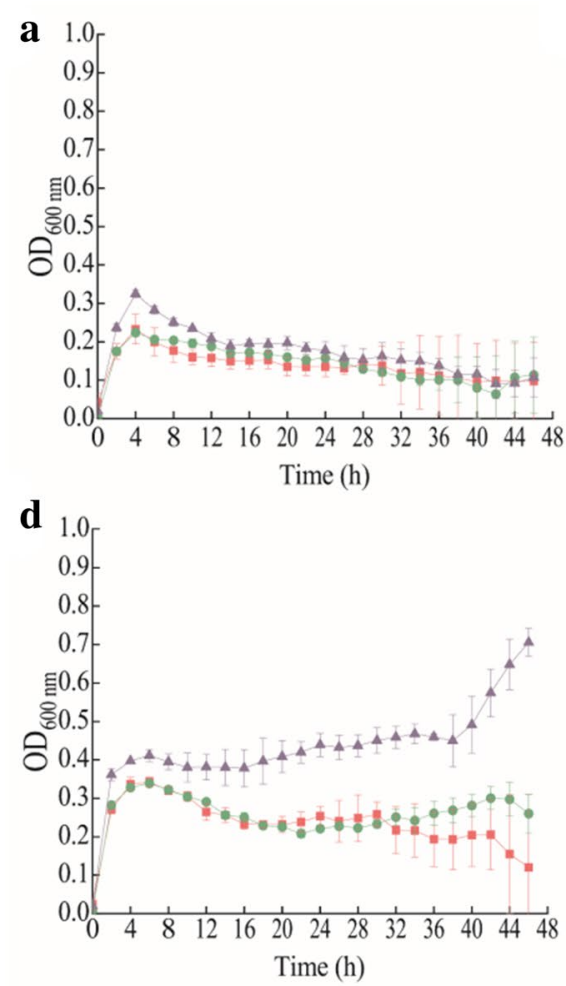
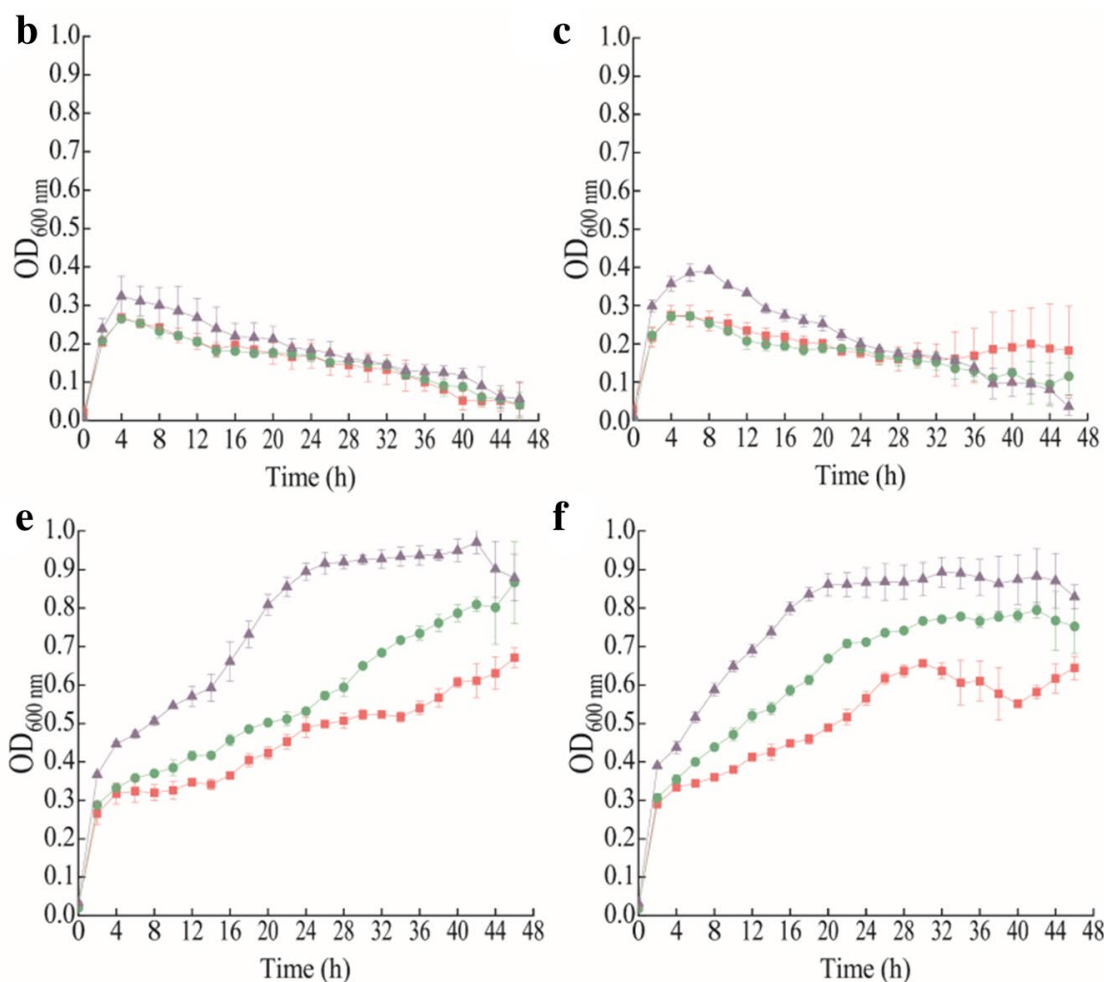

f

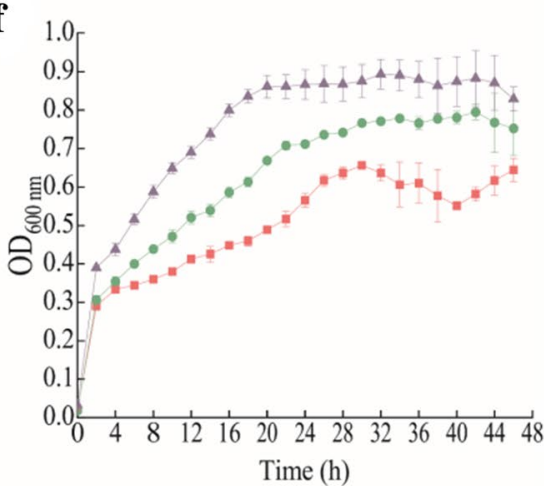

$\triangle$ WT $-\Delta V p a C h n 25 \_0734 \div-\Delta V p a C h n 25 \_0734-c o m$

Fig. 2 Survival of the WT, $\Delta V p a C h n 25 \_0734$ (543-bp), and $\Delta V p a C h n 25 \_0734-c o m$ (543-bp) strains in different pH conditions. a-f pH 5.5 (a), pH 6.0 (b), pH 6.5 (c), pH $7.0(\mathbf{d}), \mathrm{pH} 7.5(\mathbf{e})$, and $\mathrm{pH} 8.0$ (f), respectively 


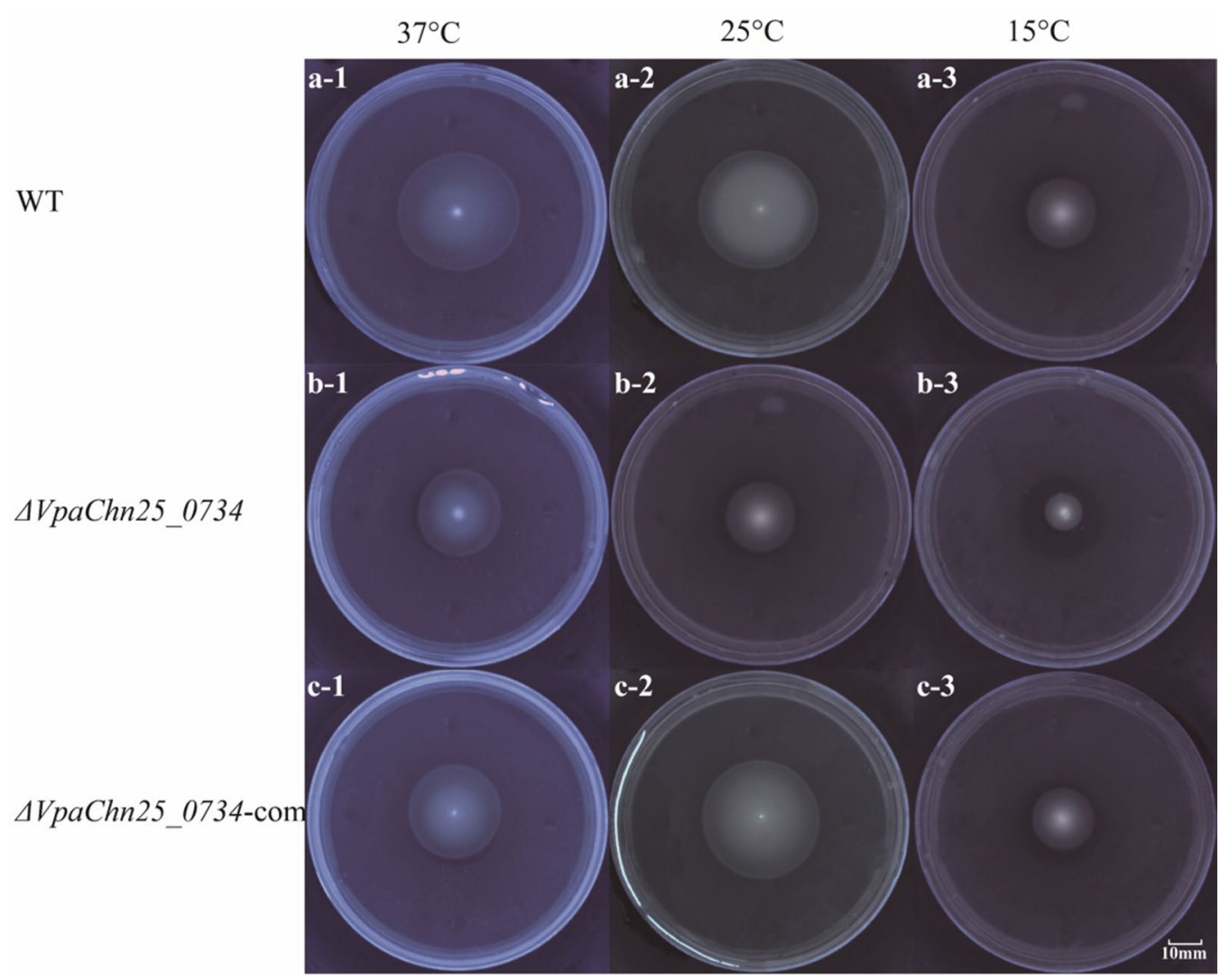

Fig. 3 Swimming mobility of the WT, $\Delta V p a C h n 25 \_0734$ (543-bp), and $\Delta V p a C h n 25 \_0734$-com (543-bp) strains at different temperatures. a-c $37^{\circ} \mathrm{C}(\mathbf{a}-1-\mathbf{c}-1), 25^{\circ} \mathrm{C}(\mathbf{a}-2-\mathbf{c}-2)$, and $15^{\circ} \mathrm{C}(\mathbf{a}-3-\mathbf{c}-\mathbf{3})$, respectively

\section{Biofilm formation of the $\Delta V p a C h n 25 \_0734$ (543-bp) mutant}

Given the growth inhibition at the lower temperatures, the dynamic process of biofilm formation of the three strains was examined at $37^{\circ} \mathrm{C}$ for $60 \mathrm{~h}$ using the crystalline violet staining method. All three strains established biofilms at three different stages (development, maturation, and diffusion) when grown in TSB medium $(\mathrm{pH} 8.5,3 \% \mathrm{NaCl})$ in static culture condition, which was consistent with the previous report (Yang et al. 2020). Nevertheless, the biofilm biomass formed by the $\Delta$ VpaChn25_0734 (543-bp) mutant was significantly less than the WT strain at development (24 h), maturation (48 h), and diffusion (60 h) stages $(p<0.05)$ (Fig. 4).

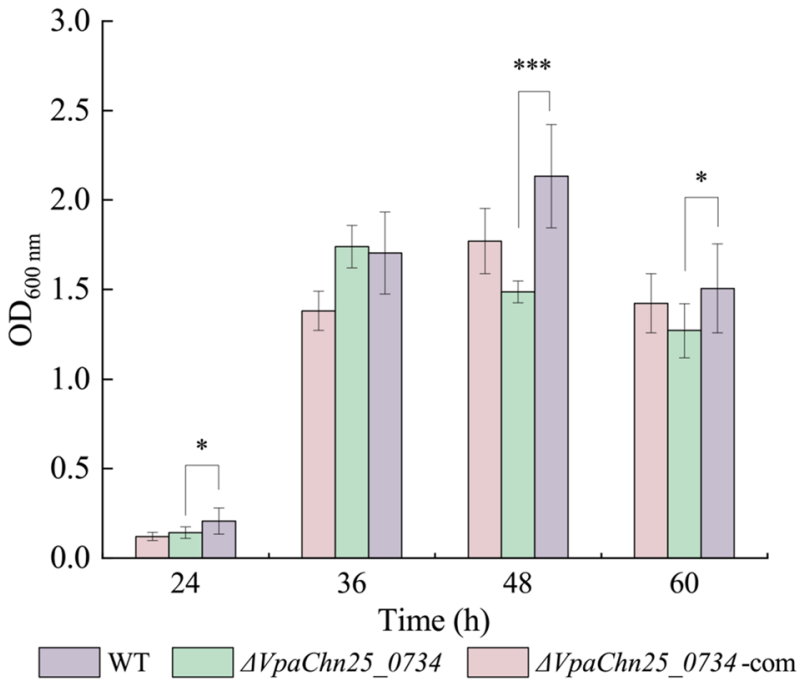

Fig. 4 Biofilm formation of the WT, $\Delta V p a C h n 25 \_0734$ (543-bp), and $\Delta$ VpaChn25_0734-com (543-bp) strains in the TSB medium at $37^{\circ} \mathrm{C}$. $* p<0.05 ; * * * p<0.001$ compared with the WT strain 

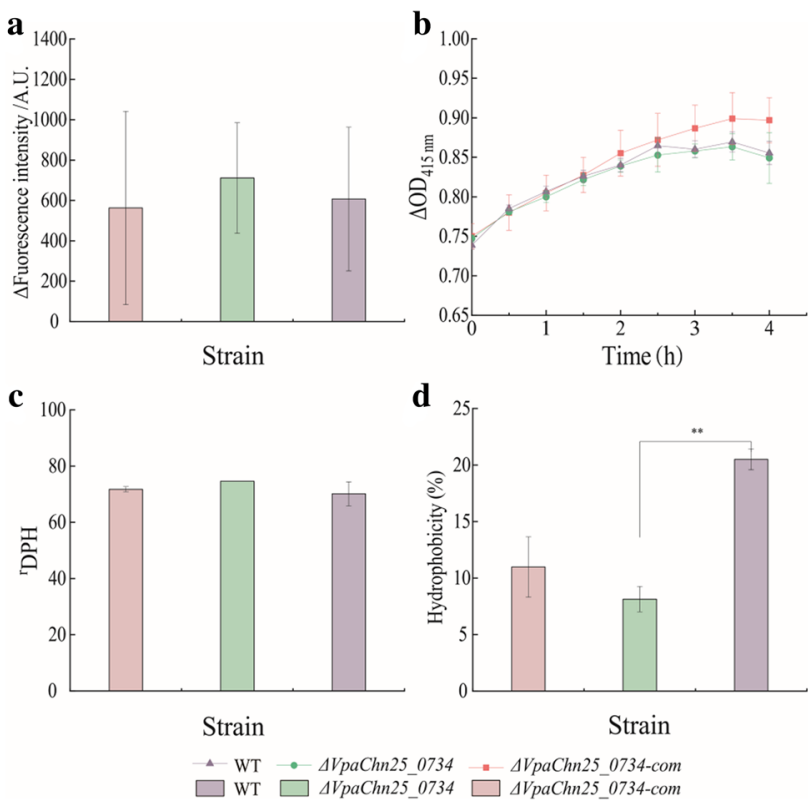

Fig. 5 Cell membrane permeability and fluidity, and surface hydrophobicity of the WT, $\Delta$ VpaChn25_0734 (543-bp), and $\Delta V p a C h n 25 \_0734$-com (543-bp) strains. a-d Outer and internal membrane permeability (a, b), fluidity (c), and hydrophobicity (d). DPH: 1,6-diphenyl-1,3,5-hexatriene. ${ }^{* *} p<0.01$ compared with the WT strain

\section{Cell membrane permeability, hydrophobicity, and fluidity of the $\Delta$ VpaChn25_0734 (543-bp) mutant}

To investigate the impact of VpaChn25_0734 (543-bp) gene deletion on cell structure of $V$. parahaemolyticus CHN25, we further examined cell membrane permeability and fluidity, and surface hydrophobicity of the three strains. As shown in Fig. 5, no significant difference in membrane permeability and fluidity was observed among the three strains grown in TSB medium $(\mathrm{pH} 8.5,3 \% \mathrm{NaCl})$ at $37{ }^{\circ} \mathrm{C}(p>0.05)$ (Fig. 5a-c). However, the cell surface hydrophobicity of the $\Delta V p a C h n 25 \_0734$ (543-bp) mutant was significantly decreased (2.52-fold) when compared to the WT $(p<0.01)$ (Fig. 5d).

\section{Secretome of the $\Delta V p a C h n 25 \_0734$ (543-bp) mutant}

The $\Delta$ VpaChn25_0734 (543-bp) mutant also grew more slowly than the WT strain when cultured in the TSB medium $\left(\mathrm{pH} 8.5,3 \% \mathrm{NaCl}\right.$ ) in static culture condition at $37^{\circ} \mathrm{C}$ (data not shown), consistent with the results of the biofilm formation experiments. As shown in Fig. 6, slightly changed secretome profiles among the three strains were observed, based on the 2D-GE analysis. Three independent parallel 2D-GE gels of each sample produced identical results (data not shown). The different protein spots were excised from the 2D-GE gels and further identified by LC-MS/MS analysis.

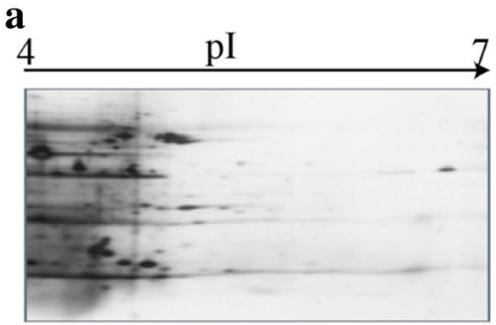

b

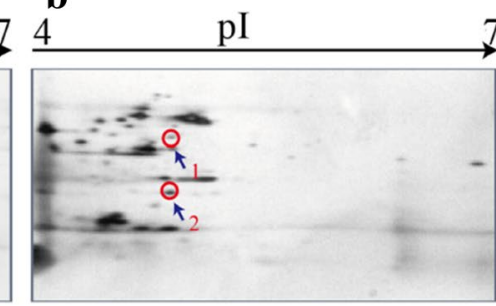

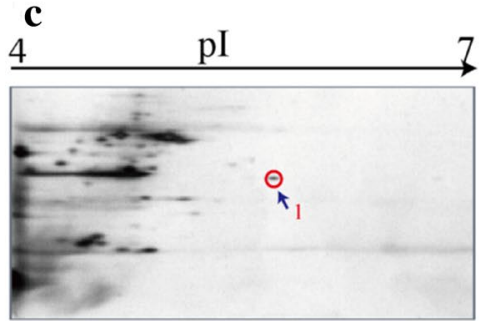

Fig. 6 The 2D-GE analysis of extracellular proteins of the WT, $\Delta V p a C h n 25 \_0734$ (543-bp), and $\Delta$ VpaChn25_0734-com (543-bp) strains. a-c WT (a); $\Delta$ VpaChn25_0734 (543-bp) (b); and $\Delta$ VpaChn25_0734-com (543-bp) (c), respectively

Table 2 Identification of the differential protein spots on the secretome profiles by LC-MS/MS analysis

\begin{tabular}{llllllcr}
\hline Protein spot & Uniprot no. & Protein & Gene & MW (Da) & pI & $\begin{array}{c}\text { Score } \\
\text { Sequence } \\
\text { coverage } \\
(\%)\end{array}$ \\
\hline 34-b-1 & A0A0D1GGT4 & 30S ribosomal protein S1 & rpsA & $61,378.44$ & 4.8 & 69.24 & 6.95 \\
$34-b-2$ & S5IPP6 & $\begin{array}{c}\text { DNA-directed RNA polymer- } \\
\text { ase subunit alpha }\end{array}$ & rpoA & $36,472.05$ & 4.78 & 76.34 & 35.15 \\
$34-c-1$ & S5IV75 & Aspartokinase & M634_16115 & $48,807.97$ & 4.98 & 60.18 & 4.89 \\
\hline
\end{tabular}



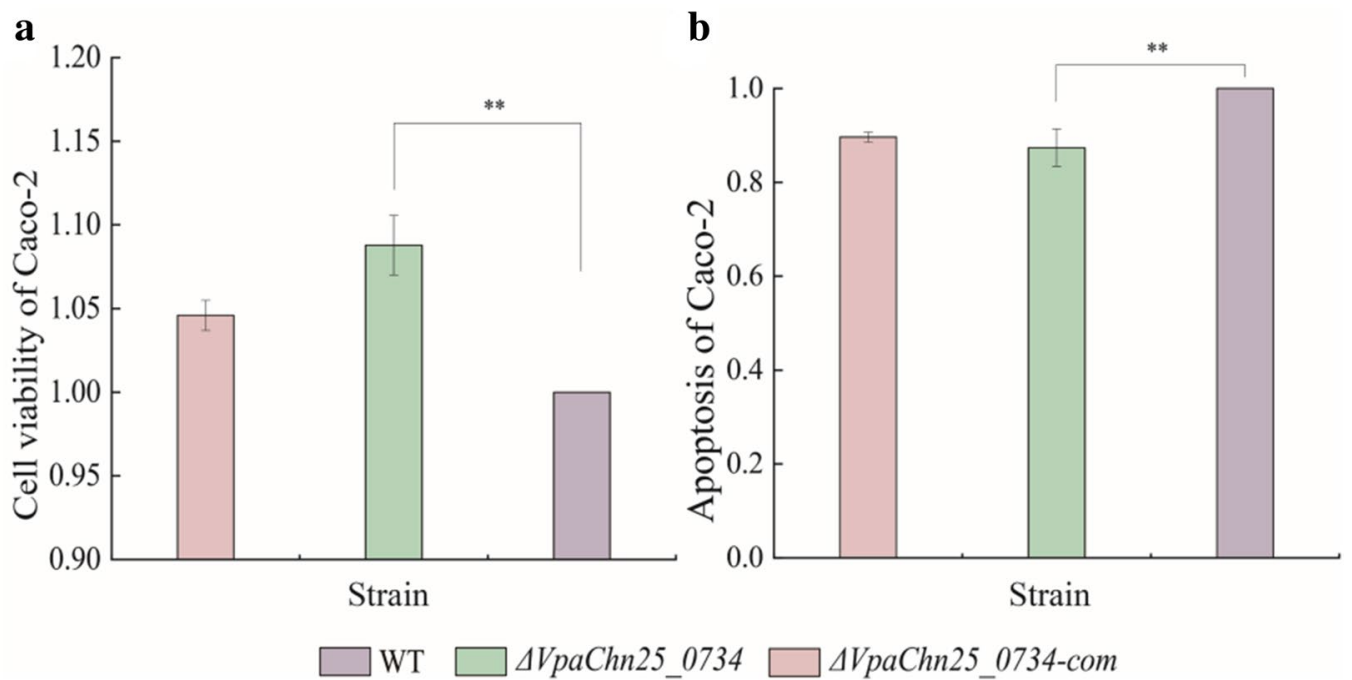

Fig. 7 The viability and apoptosis of Caco-2 cells infected by the WT, $\Delta$ VpaChn25_0734 (543-bp), and $\Delta$ VpaChn25_0734-com (543-bp) strains. (a) Cell viability; and (b) cell apoptosis. ** $p<0.01$ compared with the WT

As shown in Fig. 6, two differential extracellular proteins secreted by the $\Delta$ VpaChn25_0734 (543-bp) mutant were identified: spot 34-b-1 was identified as a 30S ribosomal protein S1 (RpsA); and Spot 34-b-2 was identified as a DNAdirected RNA polymerase subunit alpha (RpoA) (Table 2).

\section{Interaction between the $\triangle$ VpaChn25_0734 (543-bp) mutant and host intestinal epithelial cells}

As illustrated in Fig. 7, when compared to the infection with the WT strain, the viability of Caco-2 cells significantly increased 1.09-fold after infected with the $\Delta V p a C h n 25 \_0734$ (543-bp) mutant at $37^{\circ} \mathrm{C}$ for $4 \mathrm{~h}(p<0.01)$ (Fig. 7a). Meanwhile, Caco-2 cells were double-stained with membranelinked protein V-FITC and PI, and analyzed by flow cytometry. The results showed that the $\Delta V p a C h n 25 \_0734$ (543-bp) mutant induced apoptosis of Caco-2 cells at a significantly reduced rate $(0.87$-fold) than the WT strain after the infection for $4 \mathrm{~h}(p<0.01)$ (Fig. 7b). These results indicated a decrease in cytotoxicity of $V$. parahaemolyticus CHN25 in the absence of the VpaChn25_0734 (543-bp) gene.

\section{Altered transcriptome of the $\triangle V p a C h n 25 \_0734$ (543-bp) mutant}

To investigate global-level gene expression change mediated by the VpaChn25_0734 (543-bp) gene deletion, transcriptomes of the WT, $\Delta V p a C h n 25 \_0734$ (543-bp), and $\Delta$ VpaChn25_0734-com (543-bp) strains were determined, when incubated in TSB medium $(\mathrm{pH} 8.5,3 \% \mathrm{NaCl})$ at $37{ }^{\circ} \mathrm{C}$. Comparative transcriptome analysis showed that approximately $17.03 \%$ of the genes in the $\Delta V p a C h n 25 \_0734$ (543-bp) mutant were differentially expressed, which were classified into various gene functional catalogues (Fig. 8). A complete list of the DEGs in the three strains was deposited in the NCBI SRA database (http://www.ncbi.nlm.nih.gov/ sra/) under the accession number PRJNA733855. Expression of representative DEGs in the $\Delta V p a C h n 25 \_0734$ (543-bp) mutant were examined by the RT-PCR assay, and the resulting data were consistent with the transcriptomic analysis (Table S1).

\section{The major changed metabolic pathways in the $\Delta$ VpaChn25_0734 (543-bp) mutant}

Approximately thirteen significantly changed metabolic pathways were identified in the VpaChn25_0734 (543-bp) mutant, including the bacterial secretion system; terpenoid backbone biosynthesis; biofilm formation; pentose and glucuronate interconversions; fructose and mannose metabolism; purine metabolism; valine, leucine, and isoleucine degradation; pyruvate metabolism; peptidoglycan biosynthesis; valine, leucine and isoleucine biosynthesis, phosphotransferase system (PTS), two-component system (T-CS), and quorum sensing (QS) (Fig. 8).

Remarkably, approximately 81 DEGs involved in 10 of the 13 metabolic pathways (except the terpenoid backbone biosynthesis, purine metabolism, and T-CS) were all significantly down-regulated in the $\Delta V p a C h n 25 \_0734$ (543-bp) mutant $(0.051$ - to 0.500 -fold $)(p<0.05)$, when compared with the WT and $\Delta V p a C h n 25 \_0734$-com (543-bp) strains (Table 3).

In the pentose and glucuronate interconversions, expression of two DEGs were significantly repressed at the transcriptional level $(0.423-$, and 0.462 -fold $)(p<0.05)$, which encoded an L-arabinose isomerase (VpaChn25_RS23490), 


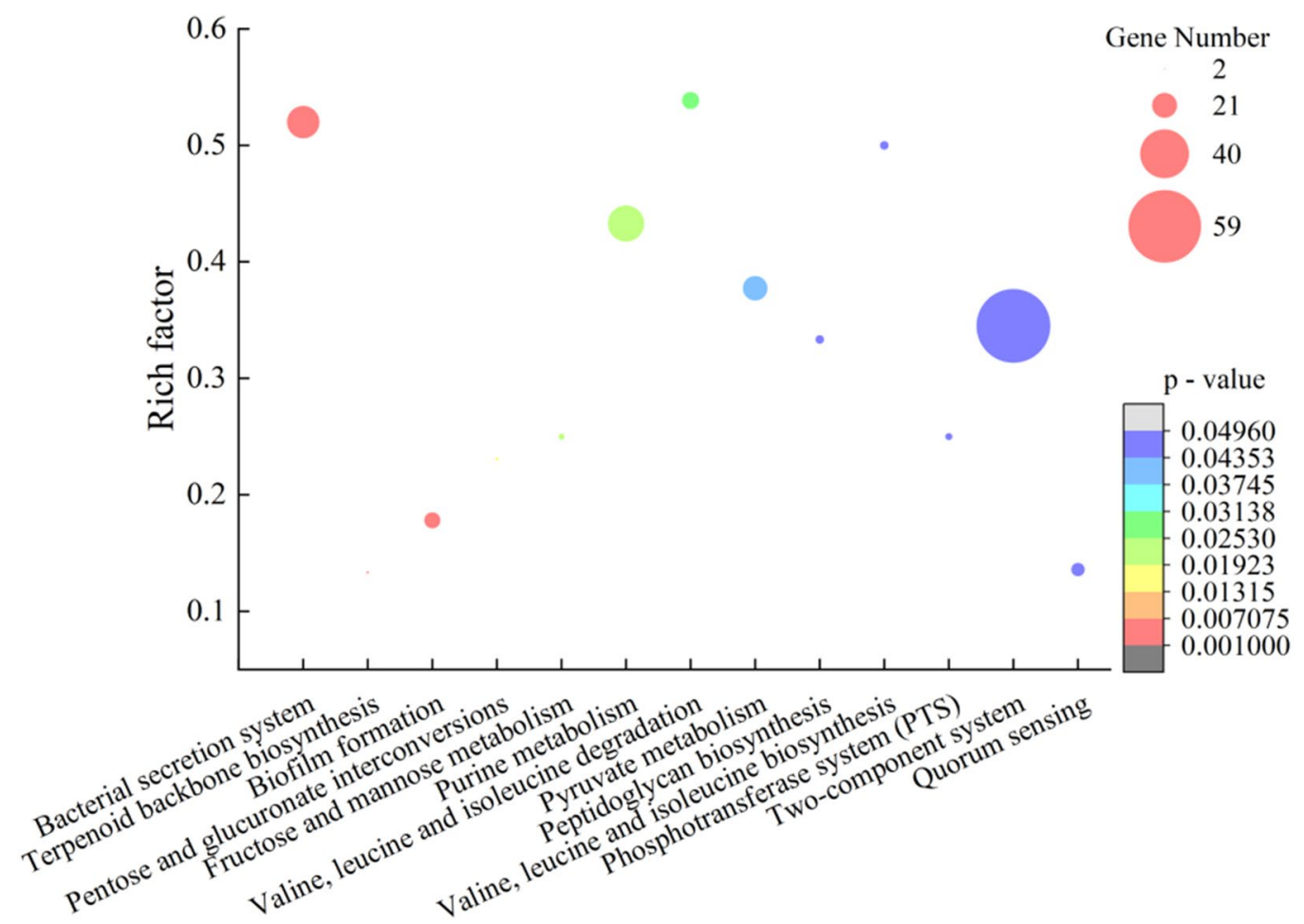

Fig. 8 Thirteen metabolic pathways significantly altered in the $\Delta$ VpaChn25_0734 (543-bp) mutant

and an oligogalacturonate lyase family protein ( $\mathrm{VpaChn} 25$ RS16200), respectively. In the fructose and mannose metabolism, 3 DEGs encoding key enzymes were also significantly inhibited (0.132- to 0.431-fold) $(p<0.05)$, including a phosphoenolpyruvate-protein phosphotransferase (VpaChn25_ RS01915), a phospho-sugar mutase (VpaChn25_RS21580), and a mannitol-1-phosphate 5-dehydrogenase (VpaChn25 RS01930). In the pyruvate metabolism, expression of 15 DGEs, e.g., a key pyruvate kinase (VpaChn25_RS10085) and a 2-hydroxyacid dehydrogenase (VpaChn25_RS16545), were significantly depressed (0.140- to 0.488 -fold). Additionally, 21 DGEs involved in T-CS were significantly downregulated (0.131- to 0.498 -fold). All these metabolic pathways were involved in carbohydrate metabolism, and the overall down-regulation trend of the DGEs sets suggested under-activity of carbon source transport and/or utilization, which may have consequently led to a certain impairment of energy supply.

In the peptidoglycan biosynthesis, expression of 6 DGEs were significantly down-regulated (0.374- to 0.498 -fold), including a phospho- $N$-acetylmuramoylpentapeptide-transferase (VpaChn25_RSO2225), a UDP- $N$-acetylmuramoyl-L-alanine-D-glutamate ligase (VpaChn25_RSO2230), a UDP- $N$-acetylmuramoyl-tripeptide-D-alanyl-D-alanine ligase (VpaChn25_RSO2220), a PBP1A family penicillin-binding protein (VpaChn25_ $R S 14065)$, a D-alanyl-D-alanine carboxypeptidase
(VpaChn25_RS23100), and a UDP- $N$-acetylmuramateL-alanine ligase (VpaChn25_RSO2245). Inhibition of the peptidoglycan biosynthetic may have compromised cell integrity and inhibited the normal growth and division of the $\Delta$ VpaChn25_0734 (543-bp) mutant.

In bacterial secretion systems, remarkably, all 13 DEGs involved in type II secretion system (T2SS) were significantly down-regulated $(0.197$ - to 0.420 -fold) $(p<0.05)$, including T2SS minor pseudopilins GspH, GspI, GspG, GspK, and GspJ (VpaChn25_RSO0645, VpaChn25_ RS00650, VpaChn25_RS00640, VpaChn25_RS00660, and VpaChn25_RSO0655); T2SS proteins M (VpaChn25_ RS00670) and GspL (VpaChn25_RS00665); T2SS ATPase GspE (VpaChn25_RS00630); T2SS inner membrane protein GspF (VpaChn25_RS00635); T2SS secretin GspD (VpaChn25_RS00625); and T2SS proteins (Vpachn25_ RS13760,Vpachn25_RS13775, and Vpachn25_RS13765). Unexpectedly, expression of 3 DGEs involved in type VI secretion system (T6SS) were enhanced (2.166- to 3.688fold) $(p<0.05$ ), including T6SS protein TssL (VpaChn25 RS20595), ATPase TssH (VpaChn25_RS20535), and lipoprotein TssJ (VpaChn25_RS20605). T6SS secrets bacterial toxins and regulates biofilm formation or stress sensing (Aschtgen et al. 2010). It was speculated that the depressed T2SS induced by the deletion of VpaChn25_0734 (543-bp) may have triggered T6SS for more support in material transport and nutrient uptake. 
Table 3 Major altered metabolic pathways in the $\Delta V$ VaChn25_0734 (543-bp) mutant

\begin{tabular}{|c|c|c|c|}
\hline Metabolic pathway & Gene ID & Fold change & Gene description \\
\hline \multirow[t]{26}{*}{ Bacterial secretion system } & VpaChn25_RS18155 & 0.188 & Protein translocase subunit $\mathrm{SecF}$ \\
\hline & VpaChn25_RS00645 & 0.197 & T2SS minor pseudopilin GspH \\
\hline & VpaChn25_RS13790 & 0.214 & $\mathrm{~T} 2 \mathrm{SS} / \mathrm{T} 6 \mathrm{SS}$ protein \\
\hline & VpaChn25_RS00650 & 0.221 & T2SS minor pseudopilin GspI \\
\hline & VpaChn25_RS08830 & 0.223 & T3SS needle filament protein $\mathrm{VscF}$ \\
\hline & VpaChn25_RS00640 & 0.266 & T2SS major pseudopilin GspG \\
\hline & VpaChn25_RS13760 & 0.287 & T2SS protein \\
\hline & VpaChn25_RS18150 & 0.303 & Protein translocase subunit SecD \\
\hline & VpaChn25_RS13775 & 0.318 & $\mathrm{~T} 2 \mathrm{SS}$ protein \\
\hline & VpaChn25_RS00670 & 0.333 & $\mathrm{~T} 2 \mathrm{SS}$ protein $\mathrm{M}$ \\
\hline & VpaChn25_RS00660 & 0.335 & T2SS minor pseudopilin GspK \\
\hline & VpaChn25_RS00655 & 0.353 & T2SS minor pseudopilin GspJ \\
\hline & VpaChn25_RS13765 & 0.360 & $\mathrm{~T} 2 \mathrm{SS}$ protein \\
\hline & VpaChn25_RS02885 & 0.365 & Preprotein translocase subunit YajC \\
\hline & VpaChn25_RS00630 & 0.392 & T2SS ATPase GspE \\
\hline & VpaChn25_RS00665 & 0.413 & $\mathrm{~T} 2 \mathrm{SS}$ protein GspL \\
\hline & VpaChn25_RS00635 & 0.418 & $\mathrm{~T} 2 \mathrm{SS}$ inner membrane protein $\mathrm{GspF}$ \\
\hline & VpaChn25_RS00625 & 0.420 & T2SS secretin GspD \\
\hline & VpaChn25_RS00470 & 0.438 & Sec-independent protein translocase subunit TatA \\
\hline & VpaChn25_RS02895 & 0.500 & Protein translocase subunit $\mathrm{SecF}$ \\
\hline & VpaChn25_RS20595 & 2.166 & T6SS protein TssL $\% 2 \mathrm{C}$ long form \\
\hline & VpaChn25_RS08730 & 2.534 & SctR familyT3SS export apparatus subunit VscR \\
\hline & VpaChn25_RS20535 & 2.837 & T6SS ATPase TssH \\
\hline & VpaChn25_RS20605 & 3.688 & T6SS lipoprotein TssJ \\
\hline & VpaChn25_RS08715 & 3.947 & T3SS central stalk protein $\mathrm{VscO}$ \\
\hline & VpaChn25_RS02080 & 4.037 & Outer membrane channel protein TolC \\
\hline \multirow[t]{2}{*}{ Terpenoid backbone biosynthesis } & VpaChn25_RS01730 & 2.392 & Octaprenyl diphosphate synthase \\
\hline & VpaChn25_RS03685 & 2.642 & 4-(cytidine 5'-diphospho)-2-C-methyl-D-erythritol kinase \\
\hline \multirow[t]{13}{*}{ Biofilm formation } & VpaChn25_RS22250 & 0.051 & Catalase \\
\hline & VpaChn25_RS01165 & 0.346 & UDP-N-acetyl-D-mannosamine dehydrogenase \\
\hline & VpaChn25_RS19800 & 0.352 & GGDEF and EAL domain-containing protein \\
\hline & VpaChn25_RS12510 & 0.359 & Porin \\
\hline & VpaChn25_RS12935 & 0.378 & Carbon storage regulator CsrA \\
\hline & VpaChn25_RS01180 & 0.406 & UDP-N-acetylglucosamine 2-epimerase (hydrolyzing) \\
\hline & VpaChn25_RS01160 & 0.422 & UDP-N-acetylglucosamine 2-epimerase (non-hydrolyzing) \\
\hline & VpaChn25_RS15210 & 0.494 & EAL domain-containing protein \\
\hline & VpaChn25_RS15250 & 0.499 & Class I adenylate cyclase \\
\hline & VpaChn25_RS01255 & 2.169 & UDP-N-acetylglucosamine 2-epimerase (non-hydrolyzing) \\
\hline & VpaChn25_RS18960 & 2.719 & Porin family protein \\
\hline & VpaChn25_RS04330 & 3.800 & Transmembrane regulator ToxS \\
\hline & VpaChn25_RS04335 & 4.405 & Transcriptional regulator ToxR \\
\hline \multirow[t]{3}{*}{ Pentose and glucuronate interconversions } & VpaChn25_RS23490 & 0.423 & $\mathrm{~L}$-arabinose isomerase \\
\hline & VpaChn25_RS16200 & 0.462 & Oligogalacturonate lyase family protein \\
\hline & VpaChn25_RS14015 & 3.101 & Ribulose-phosphate 3-epimerase \\
\hline \multirow[t]{5}{*}{ Fructose and mannose metabolism } & VpaChn25_RS01915 & 0.132 & Phosphoenolpyruvate-protein phosphotransferase \\
\hline & VpaChn25_RS21580 & 0.222 & Phospho-sugar mutase \\
\hline & VpaChn25_RS01930 & 0.431 & Mannitol-1-phosphate 5-dehydrogenase \\
\hline & VpaChn25_RS20215 & 2.537 & Mannose-6-phosphate isomerase $\% 2 \mathrm{C}$ class I \\
\hline & VpaChn25_RS01350 & 3.472 & Class II fructose-bisphosphatase \\
\hline Purine metabolism & VpaChn25_RS03475 & 0.165 & LOG family protein \\
\hline
\end{tabular}


Table 3 (continued)

\begin{tabular}{|c|c|c|c|}
\hline Metabolic pathway & Gene ID & Fold change & Gene description \\
\hline & VpaChn25_RS11920 & 0.172 & Phosphopentomutase \\
\hline & VpaChn25_RS22525 & 0.198 & Purine-nucleoside phosphorylase \\
\hline & VpaChn25_RS11915 & 0.252 & Purine-nucleoside phosphorylase \\
\hline & VpaChn25_RS01570 & 0.315 & 2'\%2C3'-cyclic-nucleotide 2'-phosphodiesterase \\
\hline & VpaChn25_RSO2805 & 0.355 & Exopolyphosphatase \\
\hline & VpaChn25_RSO3725 & 0.498 & Bifunctional UDP-sugar hydrolase/5'-nucleotidase \\
\hline & VpaChn25_RS09575 & 2.133 & Ribonucleotide-diphosphate reductase subunit beta \\
\hline & VpaChn25_RS12730 & 2.239 & Hypoxanthine phosphoribosyltransferase \\
\hline & VpaChn25_RS16050 & 2.239 & Pyrimidine/purine nucleoside phosphorylase \\
\hline & VpaChn25_RS09525 & 2.385 & Ribonucleoside-diphosphate reductase subunit alpha \\
\hline & VpaChn25_RS03680 & 2.618 & Ribose-phosphate pyrophosphokinase \\
\hline & VpaChn25_RS19630 & 2.870 & Inosine/guanosine kinase \\
\hline & VpaChn25_RS17840 & 3.332 & Adenosine deaminase \\
\hline & VpaChn25_RS06885 & 3.837 & $\begin{array}{l}\text { Formate-dependent phosphoribosylglycinamide formyltrans- } \\
\text { ferase }\end{array}$ \\
\hline & VpaChn25_RS06070 & 4.237 & Adenylosuccinate lyase \\
\hline & VpaChn25_RS03015 & 4.441 & Glutamine-hydrolyzing GMP synthase \\
\hline & VpaChn25_RS03010 & 4.645 & IMP dehydrogenase \\
\hline & VpaChn25_RS18400 & 4.849 & GMP reductase \\
\hline & VpaChn25_RS15545 & 5.336 & 5-(Carboxyamino)imidazole ribonucleotide synthase \\
\hline & VpaChn25_RS01595 & 5.550 & Adenylyl-sulfate kinase \\
\hline & VpaChn25_RSO3315 & 5.727 & Phosphoribosylformylglycinamidine synthase \\
\hline & VpaChn25_RS15540 & 6.048 & 5-(Carboxyamino)imidazole ribonucleotide mutase \\
\hline & VpaChn25_RS14820 & 6.718 & Phosphoribosylamine-glycine ligase \\
\hline & VpaChn25_RS01585 & 7.035 & Sulfate adenylyltransferase subunit CysN \\
\hline & VpaChn25_RS06715 & 7.054 & Phosphoribosylaminoimidazolesuccinocarboxamide synthase \\
\hline & VpaChn25_RS01580 & 7.268 & Sulfate adenylyltransferase subunit CysD \\
\hline & VpaChn25_RS11200 & 9.446 & Phosphoribosylformylglycinamidine cyclo-ligase \\
\hline & VpaChn25_RS14815 & 9.555 & $\begin{array}{l}\text { Bifunctional phosphoribosylaminoimidazolecarboxamide } \\
\text { formyltransferase/IMP cyclohydrolase }\end{array}$ \\
\hline \multirow[t]{14}{*}{ Valine, leucine and isoleucine degradation } & VpaChn25_RS20950 & 0.207 & Enoyl-CoA hydratase/isomerase family protein \\
\hline & VpaChn25_RS20945 & 0.240 & Methylcrotonoyl-CoA carboxylase \\
\hline & VpaChn25_RS20905 & 0.255 & 3-hydroxyisobutyrate dehydrogenase \\
\hline & VpaChn25_RS20955 & 0.279 & Hydroxymethylglutaryl-CoA lyase \\
\hline & VpaChn25_RS20940 & 0.385 & Isovaleryl-CoA dehydrogenase \\
\hline & VpaChn25_RS20925 & 0.422 & CoA-acylating methylmalonate-semialdehyde dehydrogenase \\
\hline & VpaChn25_RS22255 & 0.473 & NAD(P)-dependent oxidoreductase \\
\hline & VpaChn25_RS15655 & 0.484 & Branched-chain-amino-acid transaminase \\
\hline & VpaChn25_RS18590 & 3.000 & 3-hydroxyisobutyrate dehydrogenase \\
\hline & VpaChn25_RS18535 & 3.301 & Hydroxymethylglutaryl-CoA lyase \\
\hline & VpaChn25_RS18545 & 3.733 & Enoyl-CoA hydratase/isomerase family protein \\
\hline & VpaChn25_RS18550 & 4.207 & Methylcrotonoyl-CoA carboxylase \\
\hline & VpaChn25_RS18555 & 5.096 & Isovaleryl-CoA dehydrogenase \\
\hline & VpaChn25_RS18570 & 9.201 & CoA-acylating methylmalonate-semialdehyde dehydrogenase \\
\hline
\end{tabular}


Table 3 (continued)

\begin{tabular}{|c|c|c|c|}
\hline Metabolic pathway & Gene ID & Fold change & Gene description \\
\hline \multirow[t]{20}{*}{ Pyruvate metabolism } & VpaChn25_RS10085 & 0.140 & Pyruvate kinase \\
\hline & VpaChn25_RS22640 & 0.175 & FMN-dependent L-lactate dehydrogenase LldD \\
\hline & VpaChn25_RS16545 & 0.233 & 2-hydroxyacid dehydrogenase \\
\hline & VpaChn25_RS12875 & 0.255 & Sodium-extruding oxaloacetate decarboxylase subunit alpha \\
\hline & VpaChn25_RS10425 & 0.320 & Lactoylglutathione lyase \\
\hline & VpaChn25_RS06690 & 0.352 & NAD-dependent malic enzyme \\
\hline & VpaChn25_RS20420 & 0.360 & D-lactate dehydrogenase \\
\hline & VpaChn25_RS14690 & 0.377 & Class II fumarate hydratase \\
\hline & VpaChn25_RS12870 & 0.387 & Sodium ion-translocating decarboxylase subunit beta \\
\hline & VpaChn25_RS20930 & 0.389 & Thiolase family protein \\
\hline & VpaChn25_RS14715 & 0.400 & Acetate-CoA ligase \\
\hline & VpaChn25_RS12740 & 0.410 & Dihydrolipoyl dehydrogenase \\
\hline & VpaChn25_RS12745 & 0.414 & $\begin{array}{l}\text { Pyruvate dehydrogenase complex dihydrolipoyllysine-residue } \\
\text { acetyltransferase }\end{array}$ \\
\hline & VpaChn25_RS22970 & 0.435 & $\begin{array}{l}\text { Formate } \mathrm{C} \text {-acetyltransferase/glycerol dehydratase family glycyl } \\
\text { radical enzyme }\end{array}$ \\
\hline & VpaChn25_RS17630 & 0.488 & Phosphoenolpyruvate synthase \\
\hline & VpaChn25_RSO8880 & 2.772 & Aldehyde dehydrogenase \\
\hline & VpaChn25_RSO2855 & 5.609 & Malate synthase A \\
\hline & VpaChn25_RS17335 & 6.710 & VOC family protein \\
\hline & VpaChn25_RS18565 & 17.794 & Thiolase family protein \\
\hline & VpaChn25_RS16830 & 25.569 & Malate synthase \\
\hline \multirow[t]{7}{*}{ Peptidoglycan biosynthesis } & VpaChn25_RSO2225 & 0.374 & Phospho-N-acetylmuramoyl-pentapeptide-transferase \\
\hline & VpaChn25_RSO2230 & 0.381 & UDP-N-acetylmuramoyl-L-alanine-D-glutamate ligase \\
\hline & VpaChn25_RSO2220 & 0.394 & UDP-N-acetylmuramoyl-tripeptide-D-alanyl-D-alanine ligase \\
\hline & VpaChn25_RS14065 & 0.471 & PBP1A family penicillin-binding protein \\
\hline & VpaChn25_RS23100 & 0.493 & D-alanyl-D-alanine carboxypeptidase \\
\hline & VpaChn25_RSO2245 & 0.498 & UDP-N-acetylmuramate-L-alanine ligase \\
\hline & VpaChn25_RS23215 & 3.035 & Monofunctional biosynthetic peptidoglycan transglycosylase \\
\hline \multirow[t]{7}{*}{ Valine, leucine and isoleucine biosynthesis } & VpaChn25_RS01810 & 0.230 & 3-isopropylmalate dehydratase large subunit \\
\hline & VpaChn25_RS01820 & 0.231 & 2-isopropylmalate synthase \\
\hline & VpaChn25_RS01815 & 0.236 & 3-isopropylmalate dehydrogenase \\
\hline & VpaChn25_RS01805 & 0.278 & 3-isopropylmalate dehydratase small subunit \\
\hline & VpaChn25_RS15665 & 0.392 & Threonine ammonia-lyase $\% 2 \mathrm{C}$ biosynthetic \\
\hline & VpaChn25_RS15660 & 0.402 & Dihydroxy-acid dehydratase \\
\hline & VpaChn25_RS00150 & 0.499 & Ketol-acid reductoisomerase \\
\hline \multirow[t]{6}{*}{ Phosphotransferase system (PTS) } & VpaChn25_RS19520 & 0.180 & PTS fructose transporter subunit IIBC \\
\hline & VpaChn25_RS13650 & 0.257 & PTS IIA-like nitrogen regulatory protein PtsN \\
\hline & VpaChn25_RS13660 & 0.322 & HPr family phosphocarrier protein \\
\hline & VpaChn25_RS22265 & 0.362 & PTS fructose transporter subunit IIB \\
\hline & VpaChn25_RS22270 & 0.429 & PTS sugar transporter subunit IIA \\
\hline & VpaChn25_RS21735 & 3.677 & PTS sugar transporter subunit IIA \\
\hline \multirow[t]{3}{*}{ Two-component system } & VpaChn25_RS01005 & 0.131 & Methyl-accepting chemotaxis protein \\
\hline & VpaChn25_RS09025 & 0.162 & Response regulator \\
\hline & VpaChn25_RS21540 & 0.221 & COX15/CtaA family protein \\
\hline
\end{tabular}


Table 3 (continued)

\begin{tabular}{|c|c|c|c|}
\hline Metabolic pathway & Gene ID & Fold change & Gene description \\
\hline & VpaChn25_RSO2375 & 0.276 & $\begin{array}{l}\text { Aerobic respiration two-component sensor histidine kinase } \\
\text { ArcB }\end{array}$ \\
\hline & VpaChn25_RS13640 & 0.283 & RNA polymerase factor sigma-54 \\
\hline & VpaChn25_RS09390 & 0.291 & Response regulator transcription factor \\
\hline & VpaChn25_RS16750 & 0.319 & Sigma-54-dependent Fis family transcriptional regulator \\
\hline & VpaChn25_RS20290 & 0.341 & Anaerobic C4-dicarboxylate transporter \\
\hline & VpaChn25_RS02065 & 0.350 & Methyl-accepting chemotaxis protein \\
\hline & VpaChn25_RS18220 & 0.352 & Response regulator \\
\hline & VpaChn25_RS05680 & 0.370 & Cytochrome bd-I oxidase subunit CydX \\
\hline & VpaChn25_RS22605 & 0.376 & Methyl-accepting chemotaxis protein \\
\hline & VpaChn25_RS15910 & 0.410 & Methyl-accepting chemotaxis protein \\
\hline & VpaChn25_RS19275 & 0.425 & Response regulator \\
\hline & VpaChn25_RS09805 & 0.429 & Methyl-accepting chemotaxis protein \\
\hline & VpaChn25_RS23375 & 0.436 & Methyl-accepting chemotaxis protein \\
\hline & VpaChn25_RS06230 & 0.442 & Trimethylamine-N-oxide reductase TorA \\
\hline & VpaChn25_RSO0235 & 0.443 & $\mathrm{ABC}$ transporter permease \\
\hline & VpaChn25_RS02795 & 0.446 & Phosphate regulon sensor histidine kinase PhoR \\
\hline & VpaChn25_RS20400 & 0.472 & Methyl-accepting chemotaxis protein \\
\hline & VpaChn25_RS11070 & 0.498 & Flagellin \\
\hline & VpaChn25_RS18435 & 2.066 & Branched-chain amino acid $\mathrm{ABC}$ transporter permease \\
\hline & VpaChn25_RS19580 & 2.127 & MFS transporter \\
\hline & VpaChn25_RS08625 & 2.144 & Tripartite tricarboxylate transporter substrate-binding protein \\
\hline & VpaChn25_RS21815 & 2.171 & Efflux RND transporter periplasmic adaptor subunit \\
\hline & VpaChn25_RS17530 & 2.189 & $\mathrm{ABC}$ transporter permease \\
\hline & VpaChn25_RS21290 & 2.283 & Methyl-accepting chemotaxis protein \\
\hline & VpaChn25_RS02145 & 2.313 & Ubiquinol-cytochrome c reductase iron-sulfur subunit \\
\hline & VpaChn25_RS04780 & 2.316 & TRAP transporter small permease \\
\hline & VpaChn25_RS20970 & 2.373 & PAS domain S-box protein \\
\hline & VpaChn25_RS18440 & 2.391 & Branched-chain amino acid $\mathrm{ABC}$ transporter permease \\
\hline & VpaChn25_RS23155 & 2.442 & Polysaccharide biosynthesis tyrosine autokinase \\
\hline & VpaChn25_RS07955 & 2.487 & Cytochrome-c oxidase $\% 2 \mathrm{C}$ cbb3-type subunit I \\
\hline & VpaChn25_RS09955 & 2.525 & Polysulfide reductase NrfD \\
\hline & VpaChn25_RS18255 & 2.568 & Methyl-accepting chemotaxis protein \\
\hline & VpaChn25_RS22465 & 2.662 & Methyl-accepting chemotaxis protein \\
\hline & VpaChn25_RS12590 & 2.713 & $\mathrm{ABC}$ transporter ATP-binding protein \\
\hline & VpaChn25_RS05585 & 2.742 & Two-component system response regulator TorR \\
\hline & VpaChn25_RS21255 & 2.753 & Phosphate $\mathrm{ABC}$ transporter substrate-binding protein \\
\hline & VpaChn25_RS07030 & 3.314 & Polyamine $\mathrm{ABC}$ transporter substrate-binding protein \\
\hline & VpaChn25_RS23145 & 3.337 & Polysaccharide export protein \\
\hline & VpaChn25_RS23150 & 3.370 & Protein-tyrosine-phosphatase \\
\hline & VpaChn25_RS10335 & 3.461 & Peptide $\mathrm{ABC}$ transporter substrate-binding protein \\
\hline & VpaChn25_RS10315 & 3.653 & $\begin{array}{l}\text { Murein tripeptide/oligopeptide ABC transporter ATP-binding } \\
\text { protein OppF }\end{array}$ \\
\hline & VpaChn25_RS10320 & 3.922 & $\mathrm{ABC}$ transporter ATP-binding protein \\
\hline & VpaChn25_RS08635 & 3.799 & Tripartite tricarboxylate transporter permease \\
\hline & VpaChn25_RS07090 & 4.041 & Oligopeptide $\mathrm{ABC}$ transporter permease OppB \\
\hline & VpaChn25_RS12585 & 4.130 & $\mathrm{ABC}$ transporter ATP-binding protein \\
\hline & VpaChn25_RS20195 & 4.309 & Hexose-6-phosphate: phosphate antiporter \\
\hline & VpaChn25_RS10325 & 5.064 & Oligopeptide $\mathrm{ABC}$ transporter permease OppC \\
\hline & VpaChn25_RS12575 & 5.102 & $\mathrm{ABC}$ transporter permease \\
\hline
\end{tabular}


Table 3 (continued)

\begin{tabular}{|c|c|c|c|}
\hline Metabolic pathway & Gene ID & Fold change & Gene description \\
\hline & VpaChn25_RSO7085 & 5.558 & $\mathrm{ABC}$ transporter permease subunit \\
\hline & VpaChn25_RS12580 & 5.629 & $\mathrm{ABC}$ transporter permease \\
\hline & VpaChn25_RS10330 & 5.675 & Oligopeptide $\mathrm{ABC}$ transporter permease OppB \\
\hline & VpaChn25_RSO0555 & 12.578 & Nitrogen regulation protein $\mathrm{NR}(\mathrm{II})$ \\
\hline & VpaChn25_RS18425 & 14.069 & $\mathrm{ABC}$ transporter ATP-binding protein \\
\hline & VpaChn25_RSO0550 & 14.169 & Nitrogen regulation protein $\mathrm{NR}(\mathrm{I})$ \\
\hline & VpaChn25_RS12570 & 15.367 & $\mathrm{ABC}$ transporter substrate-binding protein \\
\hline & VpaChn25_RS00565 & 15.620 & Glutamate-ammonia ligase \\
\hline \multirow[t]{11}{*}{ Quorum sensing } & VpaChn25_RS09030 & 0.103 & Sensor histidine kinase $\mathrm{N}$-terminal domain-containing protein \\
\hline & VpaChn25_RS12840 & 0.354 & S-ribosylhomocysteine lyase \\
\hline & VpaChn25_RS18275 & 0.418 & 3-deoxy-7-phosphoheptulonate synthase AroG \\
\hline & VpaChn25_RS25650 & 0.427 & GTP cyclohydrolase II \\
\hline & VpaChn25_RS09700 & 0.447 & Aminodeoxychorismate/anthranilate synthase component II \\
\hline & VpaChn25_RS09695 & 0.485 & Anthranilate synthase component 1 \\
\hline & VpaChn25_RSO1845 & 2.207 & Long-chain fatty acid-CoA ligase \\
\hline & VpaChn25_RS07080 & 2.757 & ATP-binding cassette domain-containing protein \\
\hline & VpaChn25_RS12735 & 2.898 & Transcriptional regulator OpaR \\
\hline & VpaChn25_RS18430 & 3.288 & Long-chain fatty acid-CoA ligase \\
\hline & VpaChn25_RS07075 & 3.456 & ATP-binding cassette domain-containing protein \\
\hline
\end{tabular}

$\mathbf{a}$
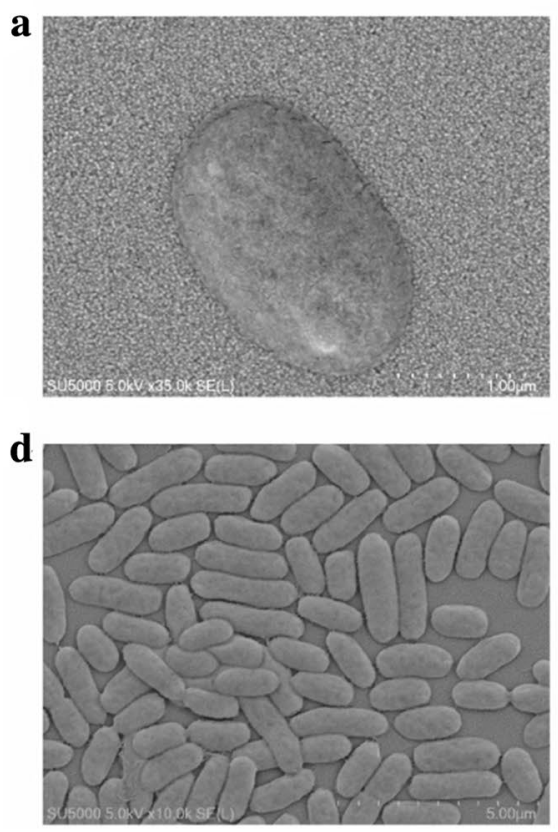

b
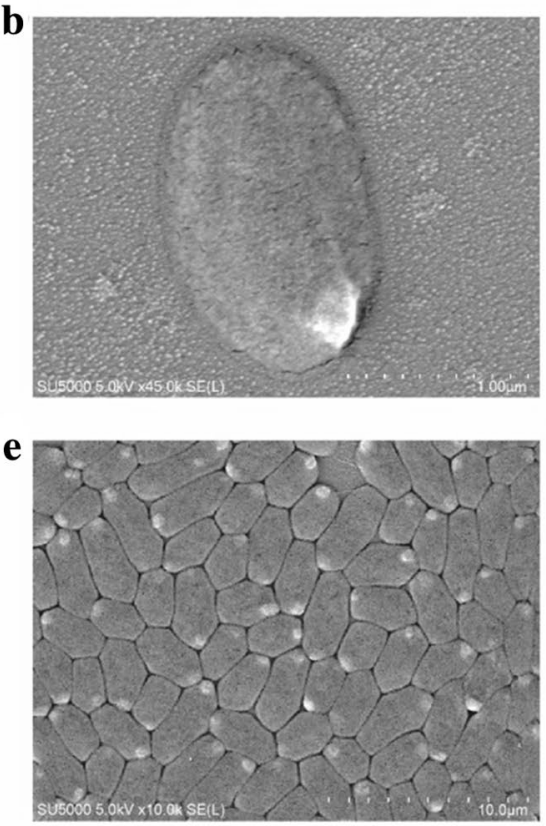
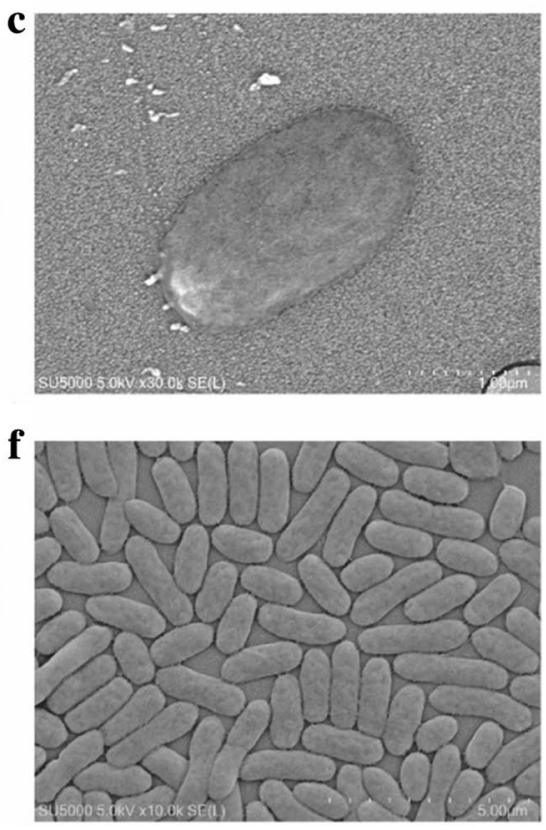

Fig. 9 The SEM observation of cell structure of the WT, $\Delta V p a C h n 25 \_0734$ (543-bp), and $\Delta V p a C h n 25 \_0734$-com (543-bp) strains. a, d WT; b, e $\Delta V p a C h n 25 \_0734$; and $\mathbf{c}, \mathbf{f} \Delta V p a C h n 25 \_0734-\mathrm{com}$

In the biofilm formation, expression of 9 DGEs, e.g., a catalase (VpaChn25_RS22250), a porin (VpaChn25_ RS12510), and a carbon storage regulator CsrA (VpaChn25_ $R S 12935)$, was significantly depressed (0.051- to 0.499 fold) $(p<0.05)$. Additionally, 6 DGEs involved in the QS
(0.103- to 0.485-fold) also showed a decrease in transcriptional levels in the $\Delta$ VpaChn25_0734 (543-bp) mutant. The SEM observation of the three strains provided cell structure evidence for the above results, in which more cytocysts were obviously present in the $\Delta$ VpaChn25_0734 (543-bp) mutant 
than the WT and $\Delta V p a C h n 25 \_0734$-com (543-bp) strains (Fig. 9).

Comparative transcriptomic analysis also revealed a few metabolic pathways that were significantly up-regulated in the $\Delta$ VpaChn25_0734 (543-bp) mutant $(p<0.05)$, such as purine metabolism, and terpenoid backbone biosynthesis. Moreover, there were several DEGs with greatly changed transcription levels (12.578- to 25.569-fold) mediated by the VpaChn25_0734 (543-bp) gene deletion. For example, expression of 22 DEGs in the purine metabolism was significantly up-regulated $(2.133$ - to 9.555 -fold $)(p<0.05)$. Additionally, a highly up-regulated gene VpaChn25_RS18565 (17.794-fold) coded for a thiolase that catalyzes the reverse Claisen condensation reaction, the first step of the biosynthesis of sterols and ketone bodies in lipid metabolism (Anbazhagan et al. 2014). The gene (VpaChn25_RS16830, 25.569-fold) encoding a malate synthase, a key enzyme responsible for malic acid synthesis in the glyoxylate cycle (Pua et al. 2003), was also greatly up-regulated. Taken, these DEGs elicited by the deletion of VpaChn25_0734 (543-bp) likely impacted multiple metabolic pathways and consequently resulted in the defective phenotypes of the $\Delta$ VpaChn25_0734 (543-bp) mutant.

\section{Distribution of the VpaChn25_0734 (543-bp) gene in bacteria}

A total of $119 \mathrm{~V}$. parahaemolyticus isolates from aquatic products collected in Shanghai, China (Su and Chen 2020) were tested for the VpaChn25_0734 (543-bp) gene by the PCR assay. The results revealed that approximately $0.84 \%(n=1)$ of the V. parahaemolyticus isolates carried the VpaChn25_0734 (543-bp) homologue. Moreover, sequence analysis against the GenBank database showed that VpaChn25_0734 (543-bp) homologues were present in one Vibrio phage (vB_VpaM_VP-3212) (marine metagenome genome assembly, Genbank accession no.: LR700235); two Vibrio species, including Vibrio campbellii (Genbank accession no.: CP020077) and V. parahaemolyticus (Genbank accession no.: CP046787); and non-Vibrio genus Shewanella oneidensis (Genbank accession no.: CP053946). Based on these identified homologues, a phylogenetic tree was constructed (Fig. 10). This analysis revealed that the
VpaChn25_0734 (543-bp) gene was phylogenetically close to a Vc3S01_A0696 gene of V. campbellii 2013 0629003S1. $V$. campbellii is an emerging aquaculture pathogen that causes Vibriosis in farmed shrimp (Nuidate et al. 2021). These results indicated that the VpaChn25_0734 (543-bp) gene exists in $V$. parahaemolyticus population, within the Vibrio genus, and even across bacterial genera.

\section{Discussion}

Vibrio parahaemolyticus is frequently isolated from seafoods at water temperatures above $15{ }^{\circ} \mathrm{C}$ ( $\mathrm{Su}$ and Chen 2020). Huge amounts of phages in aquatic ecosystems allow genetic material to jump between strains and species (Lerminiaux and Cameron 2019). Currently, biological function of prophage-encoded genes in $V$. parahaemolyticus is not yet fully understood. In this study, one such gene VpaChn25_0734 (543-bp) encoding a predicted phage morphogenetic protein in $V$. parahaemolyticus CHN25 genome was for the first time subjected to a systematic study. We successfully constructed the $\triangle V p a C h n 25 \_0734$ (543-bp) mutant and obtained its revertant $\triangle V p a C h n 25 \_0734$-com (543-bp). Our data indicated that the deletion of VpaChn25_0734 (543-bp) not only resulted in the growth hindrance of V. parahaemolyticus CHN25, particularly at lower temperatures, but also reduced the bacterial tolerance at $\mathrm{pH} 7.0-8.5$. Flagella play a crucial role in bacterial pathogenesis linked to colonization, chemotaxis, biofilm formation, and virulence (Chevance and Hughes 2008; Erhardt 2016). Flagellar motility is one of the high-energy-utilizing cellular processes (Khan et al. 2020). In this study, our results indicated that the swimming mobility of $\Delta V p a C h n 25 \_0734$ (543-bp) was significantly inhibited at lower temperatures. Moreover, the AVpaChn25_0734 (543-bp) mutant had a defect to a certain extent in all three stages of biofilm formation. These results indicated that the prophage-encoded VpaChn25_0734 (543bp) gene amplified the environmental persistence of $V$. parahaemolyticus CHN25.

Bacteria have evolved several types of secretion systems to secrete relevant substrates and effectors for bacterial adaptation to the environment and interaction with hosts (Costa et al. 2015; Wang et al. 2021b). In this study, comparative
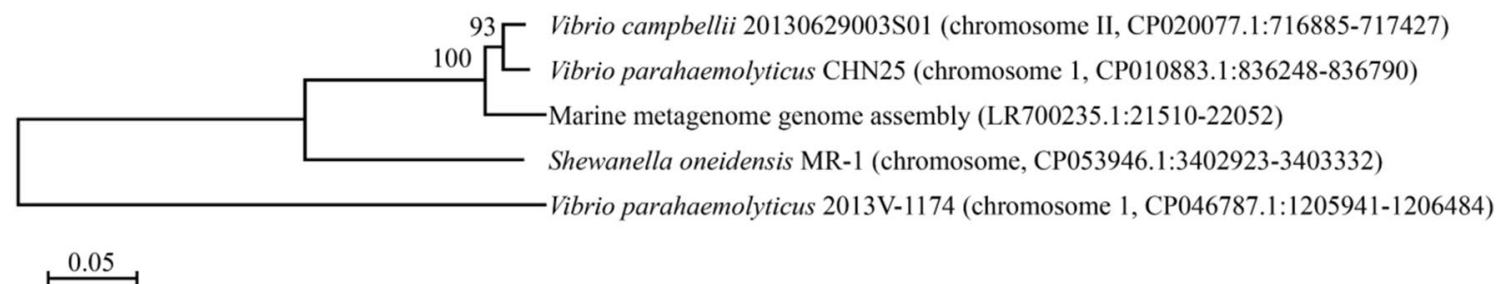

Fig. 10 Phylogenetic relationships between the VpaChn25_0734 (543-bp) gene and its homologues 
secretome analysis revealed a few differentially extracellular proteins secreted by the $\Delta$ VpaChn25_0734 (543-bp) mutant. For example, Spot 34-b-1 was identified as a 30S ribosomal protein $\mathrm{S} 1$, which is mainly involved in protein synthesis in bacteria. Despite being present intracellularly, however, it was also detected in secreted proteins of Streptococcus suis and Haemophilus parasuis (Wu et al. 2008; Wei et al. 2014). Spot 34-b-2 was a DNA-directed RNA polymerase subunit alpha protein, and its dimerization is the first step in the sequential assembly of subunits to form the holoenzyme (Degen et al. 2014). Additionally, another protein Spot 34-c-1 was identified as an aspartate kinase in the biosynthesis of the aspartate family of amino acids including methionine, threonine, and lysine (Zou et al. 2020). V. parahaemolyticus infection resulted in autophagy, cell rounding, and cell lysis, which led to proinflammatory death of infected host cells (Shimohata et al. 2011). In this study, the in vitro cell model analysis revealed that the viability of Caco-2 cells was significantly increased after the $\Delta$ VpaChn25_0734 (543bp) mutant infected at $37^{\circ} \mathrm{C}$ for $4 \mathrm{~h}$, when compared with the WT strain $(p<0.05)$. Moreover, $\Delta$ VpaChn25_0734 induced the apoptosis of Caco- 2 cells at a lower rate than WT. Taken, the differentially secreted proteins by $\triangle V p a C h n 25 \_0734$ and or the expression of VpaChn25_0734 (543-bp) gene benefited to V. parahaemolyticus $\mathrm{CHN} 25$ for its infection to the host cells.

Comparative transcriptome analysis revealed 13 significantly changed metabolic pathways in the VpaChn25_0734 (543-bp) mutant. Remarkably, all the DEGs associated with the T2SS were significantly inhibited ( 0.197 - to 0.42-fold) ( $p<0.05)$, including GspH, GspI, GspG, GspK, GspJ, GspL, GspE, GspF, GspD, Vpachn25_RS00670, Vpachn25_RS13760, Vpachn25_RS13775, Vpachn25_ $R S 13765$. The main function of bacterial T2SS is to obtain nutrients via exoproteins such as hydrolases to degrade biopolymers. T2SS also promote the secretion of toxins, adhesins, mucin, or cytochromes involved in respiration, motility, or biofilm formation (Nivaskumar and Francetic 2014; Yu et al. 2015; Matsuda et al. 2019). For example, in this study, expression of T2SS minor pseudopilin GspK (VpaChn25_RS00660) was significantly decreased (0.335fold) in the $\Delta V p a C h n 25 \_0734$ mutant. In T2SS, GspK binds on GspE N-domain via GspL and promotes GspE binding to cardiolipin to stimulate the initial rounds of ATP hydrolysis, which is required for assembly of GspG (Camberg et al. 2007; Cisneros et al. 2012). In this study, expression of GspH (Vpachn25_RS00645) was also highly repressed (0.197-fold). In T2SS, GspH binds the initiating tip complex in vitro via its globular domain, which could ensure a transition between initiation and ATPase-catalyzed elongation required for maximal efficiency of protein secretion under native conditions (Douzi et al. 2009; Korotkov and Sandkvist 2019). Similarly, three DEGs involved in the secretory-signal recognition particle (Sec-SRP) system were significantly down-regulated (0.188- to 0.5 -fold) $(p<0.05)$, including a protein translocase subunit SecF (VpaChn25_RS18155), a protein translocase subunit SecD (VpaChn25_RS18150), and a protein translocase subunit SecF (VpaChn25_RS02895). These results suggested inactive nutrient uptake and/or biofilm formation of the $\Delta$ VpaChn25_0734 (543-bp) mutant. Moreover, the DEG (VpaChn25_RS22250) encoding a catalase in the biofilm system was greatly down-regulated (0.051-fold). Catalase prevents the accumulation of hydrogen peroxide and protects cellular organelles from damage by peroxide (Goyal and Basak 2010). Additionally, the DEG (VpaChn25_RS12510) in the porin synthesis, which is a part of the biofilm system, was also decreased (0.359-fold). Porins are non-specific protein channels in bacterial outer membrane that enable the influx of hydrophilic solutes (Ades 2004; Liu et al. 2012). It will be interesting to further investigate cell location of the VpaChn25_0734 (543-bp)-encoding protein in V. parahaemolyticus CHN25 in the future research.

The PTS functions in carbohydrate transport and regulates numerous cellular processes (Schauder et al. 1998; Galinier and Deutscher 2017). In this study, comparative transcriptome data revealed that the VpaChn25_RS19520, VpaChn25_RS13650, and VpaChn25_RS22265 genes in PTS were significantly down-regulated $(0.18,0.257,0.362$ fold) in the VpaChn25_0734 (543-bp) mutant. Meanwhile, 20 DEGs involved in carbohydrate metabolism also showed significantly down-regulated transcription (0.132- to 0.488 fold). For example, the DEG encoding a pyruvate kinase (PK, VpaChn25_RS10085) in the pyruvate metabolism was highly down-regulated (0.140-fold). PK catalyzes the last step of glycolysis, in which phosphoenolpyruvate is converted to pyruvate with the production of adenosine triphosphate (ATP) (Israelsen and Vander Heiden 2015). Additionally, 21 DGEs involved in T-CS were also significantly down-regulated (0.131- to 0.498-fold). ATP-dependent $\mathrm{ABC}$ transporters actively transport molecules across the lipid membrane (Moussatova et al. 2008). The overall down-regulation trend of the DGEs sets in carbohydrate metabolism suggested that the deletion of VpaChn25_0734 (543-bp) gene negatively impacted nutrient acquisition and utilization, and consequently affected energy production and consumption of $V$. parahaemolyticus $\mathrm{CHN} 25$, consistent with defective phenotypes observed in this study.

Additionally, some DGEs were highly up-regulated in the VpaChn25_0734 (543-bp) mutant. For example, expression of an inosine monophosphate (IMP) dehydrogenase (VpaChn25_RS03010) was increased by 4.645 -fold. IMP dehydrogenase is the key enzyme in the de novo biosynthesis of guanosine monophosphate (GMP) (Salomon et al. 2004). In this study, expression of an octaprenyl diphosphate synthase (ispB, VpaChn25_RSO1730) in the terpenoid backbone 
biosynthesis was increased by 2.392 -fold, which was essential for the growth of E. coli (Okada et al. 1997; Helfrich et al. 2019). Additionally, the DEG encoding a 4-(cytidine 5'-diphospho)-2-C-methyl-D-erythritol kinase ( $\mathrm{VpaChn} 25_{-}$ $R S 03685,2.642$-fold) was also significantly up-regulated. This enzyme is essential for isoprenoid biosynthesis in some pathogenic microorganisms (Wada et al. 2003). The interaction between VpaChn25_0734 (543-bp) and these DEGs should be further investigated in future research.

\section{Conclusions}

In the present study, we characterized for the first time the prophage-encoded gene VpaChn25_0734 (543-bp) in V. parahaemolyticus CHN25 genome. The $\Delta$ VpaChn25_0734 (543-bp) mutant was obtained and the revertant $\Delta$ VpaChn25_0734-com (543-bp) was also constructed. The $\Delta$ VpaChn25_0734 (543-bp) mutant was defective in growth and swimming mobility particularly at lower temperatures, as well as in tolerance at $\mathrm{pH} 7.0-8.5$. In the absence of the VpaChn25_0734 (543-bp) gene, cell surface hydrophobicity and biofilm formation at all stages of $V$. parahaemolyticus CHN25 were significantly decreased $(p<0.05)$. These results indicated that the prophage-encoded VpaChn25_0734 (543-bp) gene amplified environmental persistence of $V$. parahaemolyticus CHN25.

Comparative secretomic analysis revealed a slightly increased extracellular proteins secreted by the $\Delta$ VpaChn25_0734 (543-bp) mutant, when compared with the WT and $\Delta$ VpaChn25_0734-com (543-bp) strains. Based on the Caco-2 cell model in vitro, the deletion of VpaChn25_0734 (543-bp) gene significantly reduced the cytotoxicity of $V$. parahaemolyticus CHN25 to human intestinal epithelial cells $(p<0.05)$, which indicated that the VpaChn25_0734 (543-bp) gene enhanced V. parahaemolyticus CHN25 fitness for surviving in the host.

Comparative transcriptome analysis revealed 13 significantly changed metabolic pathways in the $\triangle$ VpaChn25_0734 (543-bp) mutant, showing significantly down-regulated carbon source transport and/or utilization, biofilm formation, and T2SS $(p<0.05)$, consistent with its defective phenotypes. Overall, the prophage-encoded VpaChn25_0734 (543bp) gene amplified ecological persistence of $V$. parahaemolyticus CHN25. The results in this study facilitate better understanding of pathogenicity and evolution of $V$. parahaemolyticus, a leading sea foodborne pathogen worldwide.

Supplementary Information The online version contains supplementary material available at https://doi.org/10.1007/s00294-022-01229-z.
Author contributions Y.X., major experiments, data analysis, and writing-original draft; L.Y., construction of the $\Delta V p a C h n 25 \_0734-c o m$ (543-bp) revertant, phenotype and RT-PCR analysis; Y.W., construction of the $\Delta V p a C h n 25 \_0734$ (543-bp) mutant; Z.Z., secretome analysis; J.Y. supervision of the in vitro cell mode assay; S.Q. supervision, and discussion; and L.C., funding acquisition, conceptualization, and writing-review and editing. All authors have read and agreed to the published version of the manuscript.

Funding This study was supported by National Natural Science Foundation of China, Grant number 31671946; and Science and Technology Commission of Shanghai Municipal, Grant number 17050502200.

Data availability A complete list of the DEGs is available in the NCBI SRA database (http://www.ncbi.nlm.nih.gov/sra/) under the accession number PRJNA733855.

\section{Declarations}

Conflict of interest The authors declare no conflict of interest.

Ethics approval Not applicable.

Consent to participate Not applicable.

Consent for publication Shanghai Ocean University.

Open Access This article is licensed under a Creative Commons Attribution 4.0 International License, which permits use, sharing, adaptation, distribution and reproduction in any medium or format, as long as you give appropriate credit to the original author(s) and the source, provide a link to the Creative Commons licence, and indicate if changes were made. The images or other third party material in this article are included in the article's Creative Commons licence, unless indicated otherwise in a credit line to the material. If material is not included in the article's Creative Commons licence and your intended use is not permitted by statutory regulation or exceeds the permitted use, you will need to obtain permission directly from the copyright holder. To view a copy of this licence, visit http://creativecommons.org/licenses/by/4.0/.

\section{References}

Ades SE (2004) Control of the alternative sigma factor sigmaE in Escherichia coli. Curr Opin Microbiol 7:157-162. https://doi. org/10.1016/j.mib.2004.02.010

Ali S, Hossain M, Azad AB, Siddique AB, Moniruzzaman M, Ahmed MA, Amin MB, Islam MS, Rahman MM, Mondal D, Mahmud ZH (2021) Diversity of Vibrio parahaemolyticus in marine fishes of Bangladesh. J Appl Microbiol 131:2539-2551. https://doi.org/ 10.1111/jam. 15093

Anbazhagan P, Harijan RK, Kiema TR, Janardan N, Murthy MR, Michels PA, Juffer AH, Wierenga RK (2014) Phylogenetic relationships and classification of thiolases and thiolase-like proteins of Mycobacterium tuberculosis and Mycobacterium smegmatis. Tuberculosis (edinb) 94:405-412. https://doi.org/10.1016/j.tube. 2014.03.003 
Aschtgen MS, Thomas MS, Cascales E (2010) Anchoring the type VI secretion system to the peptidoglycan: TssL, TagL, TagP... what else? Virulence 1:535-540. https://doi.org/10.4161/viru.1.6.13732

Baker-Austin C, Oliver JD, Alam M, Ali A, Waldor MK, Qadri F, Martinez-Urtaza J (2018) Vibrio spp. infections. Nat Rev Dis Primers 4:8. https://doi.org/10.1038/s41572-018-0005-8

Cai Q, Zhang Y (2018) Structure, function and regulation of the thermostable direct hemolysin (TDH) in pandemic Vibrio parahaemolyticus. Microb Pathog 123:242-245. https://doi.org/10.1016/j. micpath.2018.07.021

Camberg JL, Johnson TL, Patrick M, Abendroth J, Hol WG, Sandkvist M (2007) Synergistic stimulation of EpsE ATP hydrolysis by EpsL and acidic phospholipids. EMBO J 26:19-27. https://doi. org/10.1038/sj.emboj.7601481

Cao Y, Zhang Y, Lan W, Sun X (2021) Characterization of vB_VpaP_ MGD2, a newly isolated bacteriophage with biocontrol potential against multidrug-resistant Vibrio parahaemolyticus. Arch Virol 166:413-426. https://doi.org/10.1007/s00705-020-04887-x

Castillo D, Pérez-Reytor D, Plaza N, Ramírez-Araya S, Blondel CJ, Corsini G, Bastías R, Loyola DE, Jaña V, Pavez L, García K (2018) Exploring the genomic traits of non-toxigenic Vibrio parahaemolyticus strains isolated in Southern Chile. Front Microbiol 9:161. https://doi.org/10.3389/fmicb.2018.00161

Chevance FF, Hughes KT (2008) Coordinating assembly of a bacterial macromolecular machine. Nat Rev Microbiol 6:455-465. https:// doi.org/10.1038/nrmicro1887

Cisneros DA, Bond PJ, Pugsley AP, Campos M, Francetic O (2012) Minor pseudopilin self-assembly primes type II secretion pseudopilus elongation. EMBO J 31:1041-1053. https://doi.org/10. 1038/emboj.2011.454

Costa TR, Felisberto-Rodrigues C, Meir A, Prevost MS, Redzej A, Trokter M, Waksman G (2015) Secretion systems in Gram-negative bacteria: structural and mechanistic insights. Nat Rev Microbiol 13:343-359. https://doi.org/10.1038/nrmicro3456

Degen D, Feng Y, Zhang Y, Ebright KY, Ebright YW, Gigliotti M, Vahedian-Movahed H, Mandal S, Talaue M, Connell N, Arnold E, Fenical W, Ebright RH (2014) Transcription inhibition by the depsipeptide antibiotic salinamide A. Elife 3:e02451. https://doi. org/10.7554/eLife.02451

Douzi B, Durand E, Bernard C, Alphonse S, Cambillau C, Filloux A, Tegoni M, Voulhoux R (2009) The XcpV/GspI pseudopilin has a central role in the assembly of a quaternary complex within the T2SS pseudopilus. J Biol Chem 284:34580-34589. https://doi. org/10.1074/jbc.M109.042366

Elmahdi S, DaSilva LV, Parveen S (2016) Antibiotic resistance of Vibrio parahaemolyticus and Vibrio vulnificus in various countries: a review. Food Microbiol 57:128-134. https://doi.org/10. 1016/j.fm.2016.02.008

Erhardt M (2016) Strategies to block bacterial pathogenesis by interference with motility and chemotaxis. Curr Top Microbiol Immunol 398:185-205. https://doi.org/10.1007/82_2016_493

Freitas C, Glatter T, Ringgaard S (2020) The release of a distinct cell type from swarm colonies facilitates dissemination of Vibrio parahaemolyticus in the environment. ISME J 14:230-244. https://doi. org/10.1038/s41396-019-0521-x

Fujino T, Okuno Y, Nakada D, Aoyama A, Fukai K, Mukai T, Ueho T (1953) On the bacteriological examination of shirasu-food poisoning. Med J Osaka Univ 4:299-304

Galinier A, Deutscher J (2017) Sophisticated regulation of transcriptional factors by the bacterial phosphoenolpyruvate: sugar phosphotransferase system. J Mol Biol 429:773-789. https://doi.org/ 10.1016/j.jmb.2017.02.006

Garin-Fernandez A, Wichels A (2020) Looking for the hidden: characterization of iysogenic phages in potential pathogenic Vibrio species from the north sea. Mar Genom 51:100725. https://doi. org/10.1016/j.margen.2019.100725
Garin-Fernandez A, Glöckner FO, Wichels A (2020) Genomic characterization of filamentous phage vB_VpaI_VP-3218, an inducible prophage of Vibrio parahaemolyticus. Mar Genomics 53:100767. https://doi.org/10.1016/j.margen.2020.100767

Ghenem L, Elhadi N, Alzahrani F, Nishibuchi M (2017) Vibrio parahaemolyticus: a review on distribution, pathogenesis, virulence determinants and epidemiology. Saudi J Med Med Sci 5:93-103. https://doi.org/10.4103/sjmms.sjmms_30_17

Goyal MM, Basak A (2010) Human catalase: looking for complete identity. Protein Cell 1:888-897. https://doi.org/10.1007/ s13238-010-0113-Z

He Y, Wang H, Chen L (2015) Comparative secretomics reveals novel virulence-associated factors of Vibrio parahaemolyticus. Front Microbiol 6:707. https://doi.org/10.3389/fmicb.2015.00707

Helfrich EJN, Lin GM, Voigt CA, Clardy J (2019) Bacterial terpene biosynthesis: challenges and opportunities for pathway engineering. Beilstein J Org Chem 15:2889-2906. https://doi.org/10.3762/ bjoc. 15.283

Huang B, Liu X, Li Z, Zheng Y, Yeung K, Cui Z, Liang Y, Zhu S, Wu S (2021) Rapid bacteria capturing and killing by AgNPs/N$\mathrm{CD}^{\circledR} \mathrm{ZnO}$ hybrids strengthened photo-responsive xerogel for rapid healing of bacteria-infected wounds. Chem Eng J 414:128805. https://doi.org/10.1016/j.cej.2021.128805

Israelsen WJ, Vander Heiden MG (2015) Pyruvate kinase: function, regulation and role in cancer. Semin Cell Dev Biol 43:43-51. https://doi.org/10.1016/j.semcdb.2015.08.004

Karan S, Choudhury D, Dixit A (2021) Immunogenic characterization and protective efficacy of recombinant CsgA, major subunit of curli fibers, against Vibrio parahaemolyticus. Appl Microbiol Biotechnol 105:599-616. https://doi.org/10.1007/ s00253-020-11038-4

Khan F, Tabassum N, Anand R, Kim YM (2020) Motility of Vibrio spp.: regulation and controlling strategies. Appl Microbiol Biotechnol 104:8187-8208. https://doi.org/10.1007/ s00253-020-10794-7

Korotkov KV, Sandkvist M (2019) Architecture, function, and substrates of the Type II secretion system. EcoSal plus. https://doi. org/10.1128/ecosalplus.ESP-0034-2018

Kumar S, Stecher G, Li M, Knyaz C, Tamura K (2018) MEGA X: molecular evolutionary genetics analysis across computing platforms. Mol Biol Evol 35:1547-1549. https://doi.org/10.1093/ molbev/msy096

Leoni F, Talevi G, Masini L, Ottaviani D, Rocchegiani E (2016) Trh $\left(t d h / t r h^{+}\right)$gene analysis of clinical, environmental and food isolates of Vibrio parahaemolyticus as a tool for investigating pathogenicity. Int J Food Microbiol 225:43-53. https://doi.org/ 10.1016/j.ijfoodmicro.2016.02.016

Lerminiaux NA, Cameron ADS (2019) Horizontal transfer of antibiotic resistance genes in clinical environments. Can J Microbiol 65:34-44. https://doi.org/10.1139/cjm-2018-0275

Letchumanan V, Chan KG, Lee LH (2014) Vibrio parahaemolyticus: a review on the pathogenesis, prevalence, and advance molecular identification techniques. Front Microbiol 5:705. https://doi.org/ 10.3389/fmicb.2014.00705

Li Y, Xie T, Pang R, Wu Q, Zhang J, Lei T, Xue L, Wu H, Wang J, Ding Y, Chen M, Wu S, Zeng H, Zhang Y, Wei X (2020) Foodborne Vibrio parahaemolyticus in China: prevalence, antibiotic susceptibility, and genetic characterization. Front Microbiol 11:1670. https://doi.org/10.3389/fmicb.2020.01670

Liu P, Li P, Jiang X, Bi D, Xie Y, Tai C, Deng Z, Rajakumar K, Ou HY (2012) Complete genome sequence of Klebsiella pneumoniae subsp. pneumoniae HS11286, a multidrug-resistant strain isolated from human sputum. J Bacteriol 194:1841-1842. https://doi.org/ 10.1128/jb.00043-12

Lu S, Wang J, Chitsaz F, Derbyshire MK, Geer RC, Gonzales NR, Gwadz M, Hurwitz DI, Marchler GH, Song JS, Thanki N, 
Yamashita RA, Yang M, Zhang D, Zheng C, Lanczycki CJ, Marchler-Bauer A (2020) CDD/SPARCLE: the conserved domain database in 2020. Nucleic Acids Res 48:d265-d268. https://doi. org/10.1093/nar/gkz991

Maje MD, Kaptchouang Tchatchouang CD, Manganyi MC, Fri J, Ateba CN (2020) Characterisation of Vibrio species from surface and drinking water sources and assessment of biocontrol potentials of their bacteriophages. Int J Microbiol 2020:8863370. https://doi. org/10.1155/2020/8863370

Matsuda S, Okada R, Tandhavanant S, Hiyoshi H, Gotoh K, Iida T, Kodama T (2019) Export of a Vibrio parahaemolyticus toxin by the Sec and type III secretion machineries in tandem. Nat Microbiol 4:781-788. https://doi.org/10.1038/s41564-019-0368-y

Meparambu Prabhakaran D, Ramamurthy T, Thomas S (2020) Genetic and virulence characterisation of Vibrio parahaemolyticus isolated from Indian coast. BMC Microbiol 20:62. https://doi.org/ 10.1186/s12866-020-01746-2

Moussatova A, Kandt C, O'Mara ML, Tieleman DP (2008) ATP-binding cassette transporters in Escherichia coli. Biochim Biophys Acta 1778:1757-1771. https://doi.org/10.1016/j.bbamem.2008. 06.009

Nivaskumar M, Francetic O (2014) Type II secretion system: a magic beanstalk or a protein escalator. Biochim Biophys Acta 1843:1568-1577. https://doi.org/10.1016/j.bbamcr.2013.12.020

Nuidate T, Kuaphiriyakul A, Surachat K, Mittraparp-Arthorn P (2021) Induction and genome analysis of HY01, a newly reported prophage from an emerging shrimp pathogen Vibrio campbellii. Microorganisms 9:400. https://doi.org/10.3390/microorganisms9 020400

Okada K, Minehira M, Zhu X, Suzuki K, Nakagawa T, Matsuda H, Kawamukai M (1997) The ispB gene encoding octaprenyl diphosphate synthase is essential for growth of Escherichia coli. J Bacteriol 179:3058-3060. https://doi.org/10.1128/jb.179.9.3058-3060. 1997

Pua EC, Chandramouli S, Han P, Liu P (2003) Malate synthase gene expression during fruit ripening of Cavendish banana (Musa acuminata cv. Williams). J Exp Bot 54:309-316. https://doi.org/ 10.1093/jxb/erg030

Salomon S, Felk A, Schäfer W (2004) 4-genomics of Candida albicans. In: Arora DK, Khachatourians GG (eds) Applied mycology and biotechnology. Elsevier, New York, pp 99-135

Santos LO, de Lanna CA, Arcanjo A, Bisch PM, von Krüger WMA (2021) Genotypic diversity and pathogenic potential of clinical and environmental Vibrio parahaemolyticus isolates from Brazil. Front Microbiol 12:602653. https://doi.org/10.3389/fmicb.2021. 602653

Schauder S, Nunn RS, Lanz R, Erni B, Schirmer T (1998) Crystal structure of the IIB subunit of a fructose permease (IIBLev) from Bacillus subtilis. J Mol Biol 276:591-602. https://doi.org/10. 1006/jmbi.1997.1544

Shimohata T, Nakano M, Lian X, Shigeyama T, Iba H, Hamamoto A, Yoshida M, Harada N, Yamamoto H, Yamato M, Mawatari K, Tamaki T, Nakaya Y, Takahashi A (2011) Vibrio parahaemolyticus infection induces modulation of IL-8 secretion through dual pathway via VP1680 in Caco-2 cells. J Infect Dis 203:537-544. https://doi.org/10.1093/infdis/jiq070

Song Y, Yu P, Li B, Pan Y, Zhang X, Cong J, Zhao Y, Wang H, Chen L (2013) The mosaic accessory gene structures of the SXT/R391like integrative and conjugative elements derived from Vibrio spp. isolated from aquatic products and environment in the Yangtze River Estuary. China. BMC Microbiol 13:214. https://doi.org/10. 1186/1471-2180-13-214

Su C, Chen L (2020) Virulence, resistance, and genetic diversity of Vibrio parahaemolyticus recovered from commonly consumed aquatic products in Shanghai. China. Mar Pollut Bull 160:111554. https://doi.org/10.1016/j.marpolbul.2020.111554
Sun X, Liu T, Peng X, Chen L (2014) Insights into Vibrio parahaemolyticus CHN25 response to artificial gastric fluid stress by transcriptomic analysis. Int J Mol Sci 15:22539-22562. https://doi. org/10.3390/ijms 151222539

Tan CW, Rukayadi Y, Hasan H, Abdul-Mutalib NA, Jambari NN, Hara H, Thung TY, Lee E, Radu S (2021) Isolation and characterization of six Vibrio parahaemolyticus lytic bacteriophages from seafood samples. Front Microbiol 12:616548. https://doi.org/10. 3389/fmicb.2021.616548

Wada T, Kuzuyama T, Satoh S, Kuramitsu S, Yokoyama S, Unzai S, Tame JR, Park SY (2003) Crystal structure of 4-(cytidine 5'-diphospho)-2-C-methyl-D-erythritol kinase, an enzyme in the non-mevalonate pathway of isoprenoid synthesis. J Biol Chem 278:30022-30027. https://doi.org/10.1074/jbc.M304339200

Walker CLF, Rudan I, Liu L, Nair H, Theodoratou E, Bhutta ZA, O'Brien KL, Campbell H, Black RE (2013) Global burden of childhood pneumonia and diarrhoea. Lancet 381:1405-1416. https://doi.org/10.1016/s0140-6736(13)60222-6

Wang LP, Zhou SX, Wang X, Lu QB, Shi LS, Ren X, Zhang HY, Wang YF, Lin SH, Zhang CH, Geng MJ, Zhang XA, Li J, Zhao SW, Yi ZG, Chen X, Yang ZS, Meng L, Wang XH, Liu YL, Cui AL, Lai SJ, Liu MY, Zhu YL, Xu WB, Chen Y, Wu JG, Yuan ZH, Li MF, Huang LY, Li ZJ, Liu W, Fang LQ, Jing HQ, Hay SI, Gao GF, Yang WZ (2021a) Etiological, epidemiological, and clinical features of acute diarrhea in China. Nat Commun 12:2464. https:// doi.org/10.1038/s41467-021-22551-Z

Wang T, Du X, Ji L, Han Y, Dang J, Wen J, Wang Y, Pu Q, Wu M, Liang H (2021b) Pseudomonas aeruginosa T6SS-mediated molybdate transport contributes to bacterial competition during anaerobiosis. Cell Rep 35:108957. https://doi.org/10.1016/j.celrep.2021.108957

Wang Z, Qin Q, Zheng Y, Li F, Zhao Y, Chen GQ (2021c) Engineering the permeability of Halomonas bluephagenesis enhanced its chassis properties. Metab Eng 67:53-66. https://doi.org/10.1016/j. ymben.2021.05.010

Wei X, Cao S, Zhang L, Wen X, Wen Y, Wu R, Huang X, Huang Y, Yan Q (2014) Comparative proteome analysis of the extracellular proteins of two Haemophilus parasuis strains Nagasaki and SW114. Biochem Biophys Res Commun 446:997-1001. https:// doi.org/10.1016/j.bbrc.2014.03.049

Wendling CC, Refardt D, Hall AR (2021) Fitness benefits to bacteria of carrying prophages and prophage-encoded antibiotic-resistance genes peak in different environments. Evolution 75:515-528. https://doi.org/10.1111/evo.14153

Wu Z, Zhang W, Lu C (2008) Comparative proteome analysis of secreted proteins of Streptococcus suis serotype 9 isolates from diseased and healthy pigs. Microb Pathog 45:159-166. https://doi. org/10.1016/j.micpath.2008.04.009

Yang L, Wang Y, Yu P, Ren S, Zhu Z, Jin Y, Yan J, Peng X, Chen L (2020) Prophage-related gene VpaChn25_0724 contributes to cell membrane integrity and growth of Vibrio parahaemolyticus CHN25. Front Cell Infect Microbiol 10:595709. https://doi.org/ 10.3389/fcimb.2020.595709

Yu Y, Fang L, Zhang Y, Sheng H, Fang W (2015) VgrG2 of type VI secretion system 2 of Vibrio parahaemolyticus induces autophagy in macrophages. Front Microbiol 6:168. https://doi.org/10.3389/ fmicb.2015.00168

Zhu C, Sun B, Liu T, Zheng H, Gu W, He W, Sun F, Wang Y, Yang M, Bei W, Peng X, She Q, Xie L, Chen L (2017) Genomic and transcriptomic analyses reveal distinct biological functions for cold shock proteins (VpaCspA and VpaCspD) in Vibrio parahaemolyticus $\mathrm{CHN} 25$ during low-temperature survival. BMC Genom 18:436. https://doi.org/10.1186/s12864-017-3784-5

Zhu Z, Yang L, Yu P, Wang Y, Peng X, Chen L (2020) Comparative proteomics and secretomics revealed virulence and antibiotic resistance-associated factors in Vibrio parahaemolyticus 
recovered from commonly consumed aquatic products. Front Microbiol 11:1453. https://doi.org/10.3389/fmicb.2020.01453

Zou X, Guo L, Huang L, Li M, Zhang S, Yang A, Zhang Y, Zhu L, Zhang H, Zhang J, Feng Z (2020) Pathway construction and metabolic engineering for fermentative production of $\beta$-alanine in Escherichia coli. Appl Microbiol Biotechnol 104:2545-2559. https://doi.org/10.1007/s00253-020-10359-8
Publisher's Note Springer Nature remains neutral with regard to jurisdictional claims in published maps and institutional affiliations. 